\title{
Hacking the optical diffraction limit: Review on recent developments of fluorescence nanoscopy
}

\author{
DING YiChen, XI Peng* \& REN QiuShi \\ Department of Biomedical Engineering, College of Engineering, Peking University, Beijing 100871, China
}

Received January 8, 2011; accepted February 25, 2011; published online May 12, 2011

\begin{abstract}
Subject to the diffraction limit, the resolution of conventional optical microscopy is constrained to about 200 and $500 \mathrm{~nm}$ in the lateral and axial planes, respectively. The advantage of optical microscopy in the life sciences over electronic microscopy, especially fluorescence microscopy, drives scientists to develop novel "hacks" to reach nanoscale resolutions by optical means. In this review, three aspects of the techniques are discussed: (1) lateral super-resolution; (2) axial super-resolution; (3) super-resolution in three dimensions. The principles of how the methods achieve the cross-barrier resolution are discussed, and recent advances in current techniques are described. With these methods, the use of fluorescence microscopy is growing quickly toward a new era: fluorescence nanoscopy that will reveal 2 orders of magnitude more information on cellular structure and dynamics.
\end{abstract}

optical nanoscopy, fluorescence microscopy, diffraction limit

Citation: $\quad$ Ding Y C, Xi P, Ren Q S. Hacking the optical diffraction limit: Review on recent developments of fluorescence nanoscopy. Chinese Sci Bull, 2011, 56: 1857-1876, doi: 10.1007/s11434-011-4502-3

The nature of light is an electromagnetic wave, with all properties of a wave such as interference and diffraction. In the field of Fraunhofer diffraction (far-field) spot size is limited instead of unrestricted shrinkage due to the existence of diffraction. In 1873, the German physicist Abbe [1] first indicated that the diffraction barrier could be defined as

$$
d=\frac{\lambda}{2 n \sin \alpha}
$$

where $\lambda$ represents wavelength and $n \sin \alpha$ is the numerical aperture (N.A.), which is usually less than 1.5 due to the physical limit of light propagation. This equation states that the resolving power of traditional microscopes is greater than one third of the wavelength, i.e. $d>(\lambda / 3)$. Subsequently, the English scientist Rayleigh [2] formulated the Rayleigh criterion:

$$
\delta \geqslant 0.61 \frac{\lambda}{n \sin \alpha} .
$$

The far-field optical resolution in a plane is at most nearly

\footnotetext{
*Corresponding author (email: xipeng@ @ coe.pku.edu.cn)
}

half the wavelength, i.e. $\delta \approx(\lambda / 2)$, so the lateral resolution is $200 \mathrm{~nm}$, since the wavelength of visible light ranges from 390 to $780 \mathrm{~nm}$. Additionally, the full-width at half maximum (FWHM) along the direction of propagation is given by [3]

$$
\Delta z \approx \frac{2 \lambda}{n \sin ^{2} \alpha} .
$$

Therefore the axial diffraction barrier is $\sim 500 \mathrm{~nm}$, which is almost twice the scale of typical macromolecules such as proteins.

Before the birth of fluorescence nanoscopy, several methods, such as near-field optical scanning microscopy (NSOM), atomic force microscopy (AFM), electron microscopy (EM), and scanning tunneling microscopy (STM), were used to detect information under $200 \mathrm{~nm}$. However, unlike fluorescence molecular specific tagging, these methods are insensitive to the cellular biochemical status. NSOM and AFM can only measure the surface profile; whereas EM needs a vacuum environment and STM requires the sample to be conductive for imaging, making them unsuitable for in 
vivo cellular study.

Driven by biological interests, far-field optical fluorescence nanoscopy has developed rapidly in the past decades. In particular, with the help of a novel potential two-photon fluorescent DNA probe, Zhao et al. [4] pointed out that this probe is attractive for nucleic imaging in two-photon fluorescence microscopy. Ye et al. [5], Zhang et al. [6] have been working on the living cells or single DNA molecules and several other research groups [7-9] in China have also been studying in the field of life sciences with the aid of fluorescence microscopy. In the latest 20 years, the following well-known methods: stimulated emission depletion microscopy (STED) [10-30], ground state depletion (GSD) $[10,21,31,32]$, reversible saturable optical fluorescence transitions (RESOLFT) [15,16,33,34], saturated structured illumination microscopy (SSIM)/saturated pattern excitation microscopy (SPEM) [35-38], photo activated localization microscopy (PALM) [38-47], stochastic optical reconstruction microscopy (STORM) [48-51], ground state depletionindividual molecule return microscopy (GSDIM) [16, 52-54], and point accumulation for imaging in nanoscale topography (PAINT) [55-58], all these methods have suc- cessfully broken diffraction limits based on distinct physical processes.

\section{Excitation and read-out methods in fluorescence nanoscopy}

The conventional diffraction limit states that, the lighting of a neighborhood molecule, which is within the diffraction limit, cannot be directly separated by traditional microscopy. In order to break the diffraction barrier limit of fluorescence microscopy, it is necessary for fluorescent molecules inside the diffraction-limit focal volume not to shine simultaneously, but rather to switch on and off, namely, changing from a fluorescent state (bright state) to a non-fluorescent state (dark state). Fluorescence nanoscopy can be grouped into the following three excitation and read-out modes [53]: (1) sequential switching and read-out mode; (2) structured switching and read-out mode; and (3) stochastic switching and read-out mode (Figure 1). We will briefly introduce the principles of these three modes; the detailed applications of these methods in both lateral and axial resolution enhance- (a) Sequential switching and readout (STED, GSD, RESOLFT, etc.) (c) Stochastic switching and readout

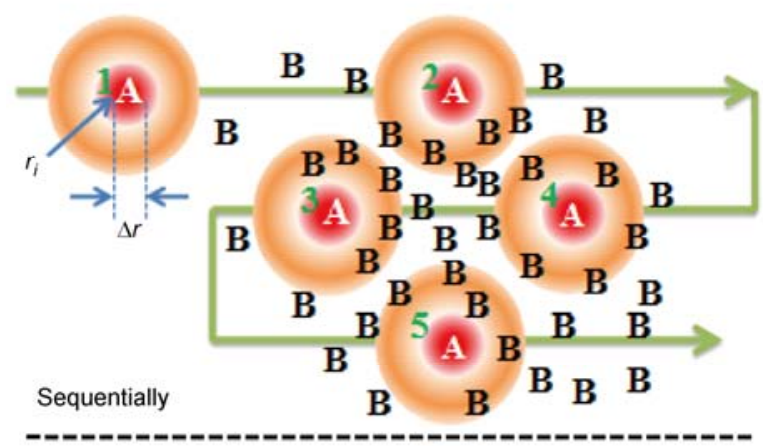

(b) Structured switching and readout (SSIM/SPEM)

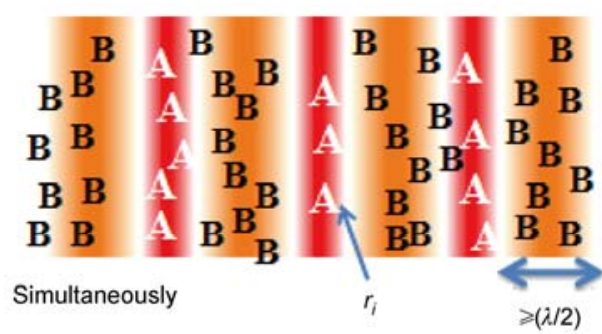

A: bright state

B: dark state (PALM, STORM, GSDIM, etc.)

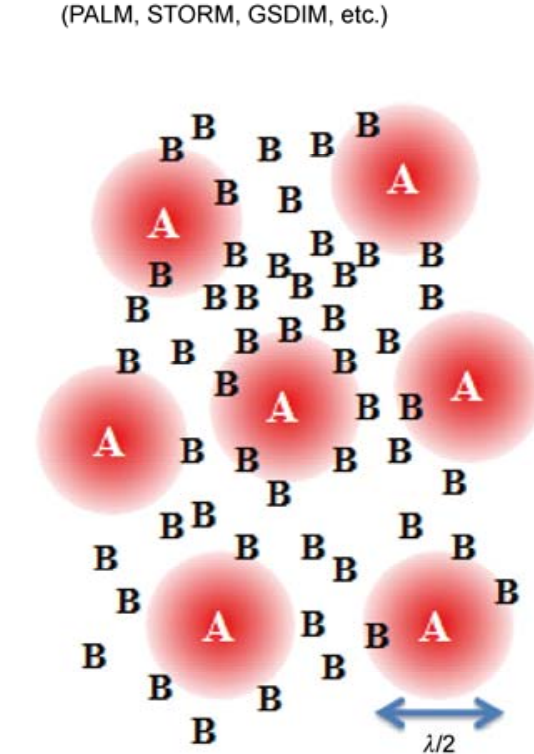

Stochastically

Figure 1 (Color online) (a) Sequential switching and read-out mode. Based on confocal microscopy, a modulated stimulating beam scans the sample spot by spot so that fluorescent molecules can be switched from a dark (B) to bright state (A). At coordinate $r_{i}$ and diameter $\Delta r$ in the field, the number of molecules detected at the same time relates to two factors: (1) scale of $\Delta r$; and (2) density of molecules in $\Delta r$. (b) Structured switching and read-out mode with a finite pattern. Using saturated illumination, only several molecules in the patterned area are excited to state A, while the rest are nonlinearly constrained in the non-fluorescent state, that is, the width of a single dark area is $\geqslant(\lambda / 2)$. (c) Stochastic switching and read-out mode. When going from state B to state A the distance between two fluorescent molecules needs to be beyond the diffraction limit to avoid overlapping. By computing the positions of $m$ photons from one molecule, the precise position of this molecule can be detected. These modes share a common trait: the molecules are switching reversibly from one state to another to realize fluorescence nanoscopy. 
ment are discussed in Sections 2-4.

\subsection{Sequential switching and read-out mode}

In the sequential switching and read-out mode, the sample is illuminated with a mechanism similar to confocal laser scanning microscopy. The main purpose of this mode is to reconstruct the point spread function (PSF) by diminishing the side lobes and shrinking the FWHM in order to strengthen the intensity of the peak through optical approaches. Since the PSF cannot be narrowed only by the excitation light source, another light source is introduced to shrink the excitation PSF via stimulated or ground state depletion. STED, GSD, and RESOLFT are the representative methods of this mode [56].

The resolution formula is modified in the following way if a non-linear saturation effect occurs [16]:

$$
d \approx \frac{\lambda}{2 n \sin \alpha \sqrt{1+a I_{\max } / I_{\mathrm{s}}}},
$$

where $I_{\max }$ is the peak intensity; $I_{\mathrm{s}}$ is the saturation intensity; $I_{\max } / I_{\mathrm{s}}$ is defined as the saturation factor $\zeta ; a$ is a parameter which is relative to the shape of the doughnut with $a>0$. One molecule in this mode has to transit between fluorescent and non-fluorescent states many times, so low power or intensity of the laser is essential to protect the fluorophore from photobleaching or photo-damage during the experiment.

\subsection{Structured switching and read-out mode}

During the process of emitting fluorescent light, the intensity of molecular emission is linearly proportional to the excitation when the excitation is relatively low. However, when the intensity of stimulation is high enough, the excitation will saturate the fluorescent molecule; therefore a nonlinear saturation effect applies. In the frequency domain, the higher-order harmonics represent the non-linear effect, whose order is related to the intensity of stimulation [35]. Therefore, the higher harmonics detected in the detection, the higher the resolution that can be theoretically achieved. Alternatively, using the on and off switching method under the condition of structured illumination, it not only improves the imaging speed, but also generates non-linear reaction when intensity is large enough [16]. Structured illumination microscopy (SIM) and the later developed SSIM/SPEM are representatives of this mode.

\subsection{Stochastic switching and read-out mode}

Stochastic switching and read-out mode differs from the former switching modes, and is based on single molecule fluorescence imaging technology. As indicated by Abbe [1], conventional far-field microscopy cannot discern separate molecules when $d \leqslant \lambda /(2 n \sin \alpha)$; however, if only one molecule appears in this field, it would be easily detected even if a barrier existed. The crucial point is to fit $m(m>>1)$ positions of photons produced from one molecule so as to locate its absolute center [53]. The highest accuracy is estimated to be $1.5 \mathrm{~nm}$ by single molecule fluorescence imaging [59]. Although single molecule fluorescence imaging technology has high precision to detect position, its read-out mode of only one molecule in an Airy disk each time is the drawback which results in a limited imaging speed [60,61].

Stochastic switching and read-out mode breaks through this bottleneck. When high density of molecules exist in an Airy disk, continuously switching the states of the fluorescent signal, transforms a high-precision position signal to a high-resolution signal in the spatial domain, and can be used to observe tiny structures. PALM (FPALM), STORM, dSTORM, GSDIM, and PAINT are all representatives of this mode [40]. By calculating the $m$ photons from the same bright molecule, the center of a fluorophore can be extracted as [53]

$$
d \approx \frac{\lambda}{2 n \sin \alpha \sqrt{m}}
$$

Note that in this mode some molecules may be positioned more precisely than others, as the number of photon emissions, $m$, approximates a Poission distribution [39]. Therefore, to ensure a certain resolution, this mode defines a brightness threshold $m>M \approx 50$. $M$ is a critical condition, depending on the average number of photon emissions in the bright state in the $X Y$-plane [16,53].

\section{Super-resolution fluorescence microscopy in the lateral plane}

\subsection{Sequential excitation and read-out mode}

(i) Stimulated emission depletion microscopy with pulse excitation. Starting in 1994, Hell et al. [17] put forward a novel method named STED for super-resolution fluorescence microscopy which was successfully demonstrated in 1999 with a spatial resolution of $106 \pm 8 \mathrm{~nm}$ by a single depletion beam [20]. The principle of this method is shown in Figure 2. $S_{0}$ represents the ground or dark state; $S_{1}$ represents the excited or bright state. Until a group of ultra-short laser pulses illuminate the sample, the molecules of the sample do not change from $S_{0}$ to $S_{1}$. From the perspective of the relaxation time and lifetime of fluorescence, the STED beam is an ultra-short, red-shifted pulse laser with a spotshape modulated to be like a donut with zero center intensity that illuminates the same focal point of a sample several picoseconds after the illumination pulse. The molecules covered by the second laser leap from $S_{1}$ to $S_{0}$ while its center still spontaneously radiates fluorescence. Therefore, the PSF is ideally confined as long as the center of ring is small enough after passing through a filter that is applied in order to eliminate other fluorescence wavelengths and illumination that are not useful for detection. Notably, neither 

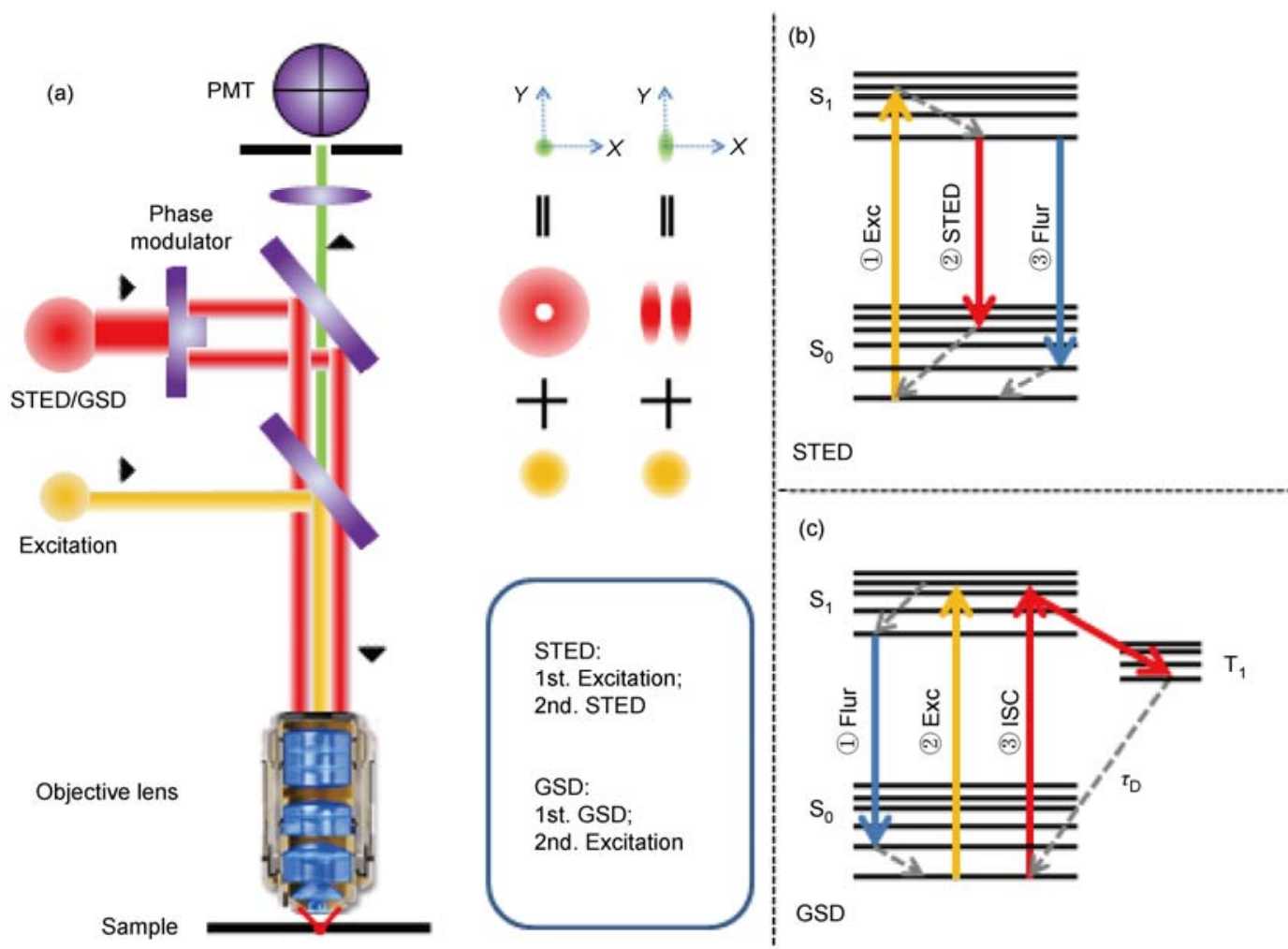

Figure 2 (Color online) (a) The equipment and principles of STED and GSD. The left panel shows a cross-section of laser or fluorescence: illumination, the STED or GSD beam and the result of superposition of the first two. In the left panel the black arrows in different directions represent the propagation of light. (b) Process of STED in both states. (c) Process of GSD in both states.

the illumination nor STED beam is beyond diffraction limit barrier. There is no obstacle to resolution as long as the depletion intensity increases $[17,18]$. This kind of "fluorescence-on-diet-method" has achieved a lateral resolution of $5.8 \pm 0.8 \mathrm{~nm}$ for a certain class of inorganic fluorophores [26], or 28 fps video-rate with a focal spot size of $62 \mathrm{~nm}$ in living neurons [27].

To reach a confined PSF, three parameters of the STED beam should be carefully chosen: wavelength, intensity, and cross-section.

(1) The wavelength of the STED beam should be longer than that of the illumination beam if it is to achieve an effective transition $S_{0} \rightarrow S_{1}$ instead of secondary excitation (note that this is also related to the absorption spectrum of the fluorescent molecule). According to Einstein's theory of stimulated emission, the STED beam has an equal probability of a stimulated absorption and emission effect on the molecules. However, because the lifetime of the ground state, $S_{0}$, is much shorter than the first-excited-state, $S_{1}$, the $\mathrm{S}_{0}$ state molecules are rapidly exhausted and are thus highly advantageous for $S_{1}$ state molecules returning to $S_{0}$ on a large scale. Thus, those molecules in the $\mathrm{S}_{0}$ state escape being stimulated to the $S_{1}$ once more.

(2) The non-linear depletion effect is the core of STED, and when it occurs the resolution formula will be modified to eq. (4) [16]. In Figure 3, when $I>>I_{\mathrm{s}}$, the non-linear sat- uration effect should be taken into consideration, and a large number of molecules are depleted from $S_{1}$ to $S_{0}$ due to the STED beam and are unable to continue fluorescent emission whose wavelength is acquired. With an increasing $\zeta$, the number of $\mathrm{S}_{1}$ molecules is severely eliminated and FWHM of PSF is substantially decreased, so that resolution will be enhanced. However, with an increase of the depletion beam,

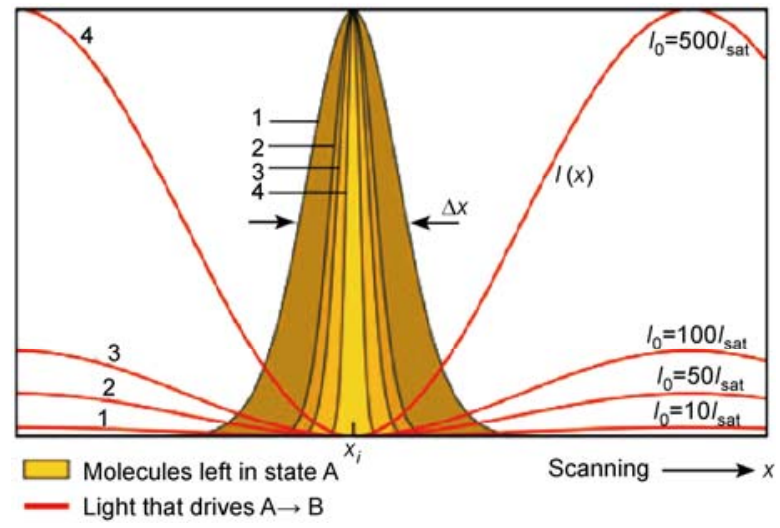

Figure 3 (Color online) The relationship between STED beam intensity and the number of molecules in the ground state. With an increasing $\zeta$, the number of $S_{1}$ molecules can be severely constrained and FWHM of PSF is substantially decreased, resulting in improvement of the resolution [18]. Image courtesy of Springer. 
photo-bleaching and photo-damage will cause rapid deterioration of the tissue and image quality will decrease due to the effect of the signal to noise ratio on the resolving power of the system. Therefore, the resolution of the system is limited by the stability of the molecules.

(3) The steeper the STED zero center region, the higher the achievable system resolution. The ring-shaped spot can deactivate fluorescence along all directions perpendicular to the propagation of light, but it is not the only choice. To meet different needs, modulating the phase of the STED beam may produce distinct cross-sections of the STED spot, so constraining FWHM in diverse directions (see Figure 2(a)), for example, the cross-sectional shrinkage can either be along one axis or all directions in the focal plane [10].

(ii) Advances in STED techniques. Typical STED relies on ultra-short pulse lasers with the same temporal delay [30]. Regardless of the complexity and cost of the experimental device in the super-resolution fluorescence imaging process, the demanding requirements of these two laser sources limit the development of STED. Hell et al. reported that a continuous wave laser source is able to replace the pulse style as long as the depletion rate of the STED beam is much greater than that of excitation $\left(k_{\text {sted }}>k_{\text {exc }}\right)$, so the time interval between illumination and depletion beams was no longer a decisive factor. The photo-reversibility of the dye molecule is a key factor in continuous wave STED (CW STED) as the switching is much more often than a pulsed STED - in fact in CW STED it is constantly switching on and off during imaging.

Similarly, compared with the former STED, CW STED also causes a depletion in proportion to power loss under the same condition of fluorescence constrained effect, but the magnitude of CW STED power is 3-5 times higher than STED power (Figure 4) [30]. Hence, to obtain the same resolution, the STED beam power has to be enlarged by a factor of $\Gamma=\tau_{\text {rep }} /\left(\tau_{\mathrm{fl}} \cdot \ln 2\right)$ [21]. In 2007, the lateral resolution of CW STED reached 29-60 nm, 5-8 times smaller than the diffraction limit, and the axial resolution obtained was $170 \mathrm{~nm}, 3.5$ times smaller than that of conventional microscopy [30].

The research on different modalities of STED has bloomed in the last 5 years. Two-photon excitation STED (TPE-STED) [22], triplet relaxation STED (T-Rex STED) [12] and STED with the aid of a reducing and oxidizing system (ROXS) [19] represent systems with less photobleaching and increased brightness. TPE-STED outperforms confocal SPE-STED in imaging the deeper layers of a scattering sample, because scattering of the excitation and fluorescence light is much less relevant in the TPE-STED case. Using a short-pulse laser for TPE and a CW laser for STED, the resolution achieves $<50 \mathrm{~nm}$ in all directions in the focal plane [22]. By stretching the interval $\Delta t$ between two sequential STED pulses, T-Rex STED improved the resolving power to $15-20 \mathrm{~nm}$ in biological samples and reduced the photo-bleaching [12]. In June, 2010, after enhancing the photo-stability of the fluorophore with the help of ROXS, STED could discern $<30 \mathrm{~nm}$ objects in the lateral direction with less photo-bleaching and more brightness [19].

The recently reported molecule orientation microscopySTED (MOM-STED) $[25,28]$ is a novel technique that dramatically simplifies the STED setup. By employing a birefringent device, MOM-STED not only aligns stimulation and STED beams in order to simplify the equipment, but also maps the molecule orientation directly in the sample, so as to open up an additional parameter for separation. Now the isotropic resolution of MOM-STED is $<40 \mathrm{~nm}$.

In 2007, two-color STED [13], by applying STED on fluorophores differing in their absorption and emission spectra, was demonstrated with a lateral resolution $<30 \mathrm{~nm}$ and $65 \mathrm{~nm}$. It indicates the viability of dual-color fluorescence STED, which is a powerful approach to image colocalization of molecular distributions.

Multi-color stimulated Raman scatting-STED (SRSSTED) $[23,24]$ using stimulated Raman scattering has been


Figure 4 (Color online) (a) In an aqueous solution of Atto647N, a continuous beam whose wavelength is $635 \mathrm{~nm}$ stimulates molecules to emit fluorescence and then a $760 \mathrm{~nm}$ wavelength STED beam depletes excited molecules. As STED power increases, the number of excited molecules decreases. Molecules come back to the ground state after depletion. (b) Comparison of CW STED power and STED power, that is, an intensity comparison. To reach the same depletion effect, CW STED power should be 3.6 times greater than that of the pulsed STED [30]. Image courtesy of Nature Publishing Group. 
suggested. From a broad spectrum ranging from 532 to 620 $\mathrm{nm}$, it can attain a resolution of $20-30 \mathrm{~nm}$ at a repetition rate of $\mathrm{MHz}$ level and expands the spectral output with multiple wavelengths in the visible range. It should be emphasized that with an all-optical resolution of $30-50 \mathrm{~nm}$ in the far-field, STED with a supercontinuum laser source achieved not only multicolor imaging but also $1 \mathrm{MHz}$ pulse repetition rates [29].

(iii) Ground state depletion. In 1995, Hell and Kroug [31] put forward a new theory which they named GSD, and an experimental device was successfully built in 2007 [10,21], while a resolution of $7.6 \mathrm{~nm}$ was achieved in 2009 [32]. A relatively long lifetime state $T_{1}$ has been employed in this method, which deactivates the number of molecules on the first-excited-state, as a triplet metastable dark state rather than exploring high laser powers. The similarity between GSD and CW STED are that they apply a continuous laser wave source, thus simplifying equipment complexity and reducing laser intensity to kilowatt per square centimeter magnitudes.

The main idea of both GSD and STED is basically to deplete the amount of saturated molecules, but the difference is that fluorophores are deactivated in distinct states.

(1) In Figure 2(c), not only $S_{0}$ and $S_{1}$ are shown to be useful in GSD method, but the figure also indicates that a triplet state, $T_{1}$, is needed. $T_{1}$ has a relatively long lifetime, $\tau_{\mathrm{T}}$, and molecules staying on the triplet state cannot transiently radiate; after $\tau_{\mathrm{T}}$ all the molecules spontaneously return to the ground state similar to the condition before stimulation. There is a certain probability, $k_{\mathrm{ISC}}$, of the occurrence of inter-system crossing between $\mathrm{T}_{1}$ and $\mathrm{S}_{1}$. Furthermore, $k_{\mathrm{ISC}}$ and $\tau_{\mathrm{T}}$ change under different experimental conditions, and therefore, through adjusting $k_{\mathrm{ISC}}$ and $\tau_{\mathrm{T}}$, the amount of molecules in $T_{1}$ is saturated, while the number of molecules in $\mathrm{S}_{0}$ is nearly zero.

(2) Different depletion channels. In STED, the beam directly stimulates fluorophores in the first-excited-state back to the ground state; in GSD, the modulated beam quickly bumps molecules in the ground state to participate in the $\mathrm{S}_{0}$ $\rightarrow \mathrm{S}_{1} \rightarrow \mathrm{S}_{0}$ cycle. During the process, a certain $k_{\mathrm{ISC}}$ probability exists in electron transition from $S_{1} \rightarrow T_{1}$ occuring. Hence, during the lifetime $\tau_{\mathrm{T}}$, as long as $\mathrm{S}_{1} \rightarrow \mathrm{T}_{1}$ transition exists and the probability $k_{\mathrm{ISC}}$ is large enough, the molecules staying in the $\mathrm{S}_{0}$ state can be intensively stimulated directly to the triplet state $T_{1}$, so the quantities of excited fluorescent molecules decrease instantly. Later on in the depletion area the stimulating beam forces the few molecules that remain in the $S_{0}$ state to fluoresce, while others are confined to the $\mathrm{T}_{1}$ state. In this way, the PSF is reduced with a low power $\mathrm{CW}$ laser.

(3) A different sequence between stimulation and depletion. Based on its special principle, stimulation $\rightarrow$ depletion by a red-shifted pulse laser is chosen in STED. Conversely, depletion $\rightarrow$ stimulation by the same wavelength laser is used in GSD. Due to the existing probability of $\mathrm{S}_{0}$ returning $\mathrm{T}_{1}$, some molecules still have the ability to emit fluorescence in the metastable state $\mathrm{T}_{1}$. This brings about invalid saturation, a broadening of the edge of the spot, and a deterioration of PSF and resolution. For instance, the fluorescent molecule Atto532 employed in GSD needs to be illuminated by a $I_{\mathrm{D}}=85 \mathrm{~kW} / \mathrm{cm}^{2}$ depletion beam for $500 \mu \mathrm{s}$, then application of a $I_{\mathrm{p}}=7 \mathrm{~kW} / \mathrm{cm}^{2}$ stimulation to excite the sample after depletion. After $60 \mathrm{~ms}$, which is the lifetime of $\mathrm{T}_{1}$, the fluorophore recovers from the transient dark state to emit fluorescence again. By careful control of the excitation rate to avoid photo-bleaching, the fluorophore can be photoswitched multiple times and behave just like fresh fluorophores [10].

Currently, GSD is mainly limited in two aspects: (a) The metastable triplet state $T_{1}$ has a close relationship with photo-bleaching, and thus, strictly controlling the stimulation intensity and frequency to make this process reversible is a fundamental necessity. (b) The lifetime $\tau_{\mathrm{T}}$ of $\mathrm{T}_{1}$ is also critical. The laser should not scan to a new spot until all the molecules return to $S_{0}$. Although a higher resolution is achieved with a longer $\tau_{\mathrm{T}}$, imaging speed will be sacrificed unless structural illumination is applied.

(iv) Reversible saturable optical fluorescence transitions. From the above three methods, the key-point of the sequential switching and read-out mode is how to deplete fluorescent molecules by non-linear saturation effects occurring between stimulation and fluorescence, in order to reversibly switch molecular states. RESOLFT represents the whole principle of this mode [15].

By forcing the excited fluorophore to transit to another transient metastable dark state, RESOLFT achieves not only the same imaging quality as STED but also reduces laser power and minimizes photo-bleaching and photo-toxicity. Hell's group seized upon this great idea hidden behind STED, and GSD to transform the physical process to a chemical one, thereby allowing the dark state condition to be manipulated [16]. In theory, without considering the impact of imaging speed, when the lifetime of the dark state is infinite, the intensity of saturated light can be infinitely small, and thus utilizes ultra-low-intensity light to change the conditions of the fluorophore [34]. Compared with previous reported techniques, high-power laser is no longer explored in RESOLFT, and the reversible photoswitch with different wavelengths illuminates photosensitive fluorescent protein (such as FP595 or Dronpa) instead. Furthermore, the novel method treats the isomeric state as $S_{1}$ and $S_{0}$ states. This technology has improved resolution by up to $50-100$ $\mathrm{nm}$ in the focal plane [33].

\subsection{Structured illumination microscopy}

(i) Saturated structured illumination microscopy/saturated pattern excitation microscopy. The optical transfer function (OTF) is the Fourier transform of the PSF. When the side lobe of the PSF is low enough, the Rayleigh criterion 
(a)

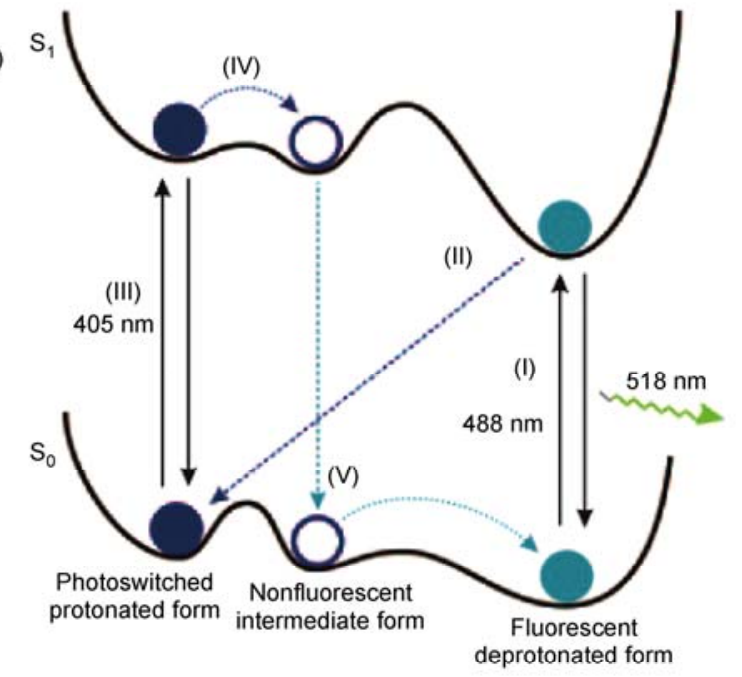

(b)



(c)

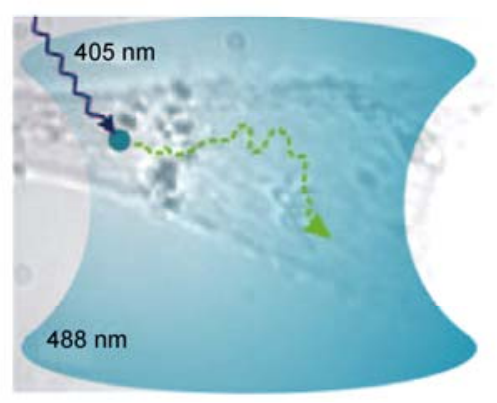

(d)

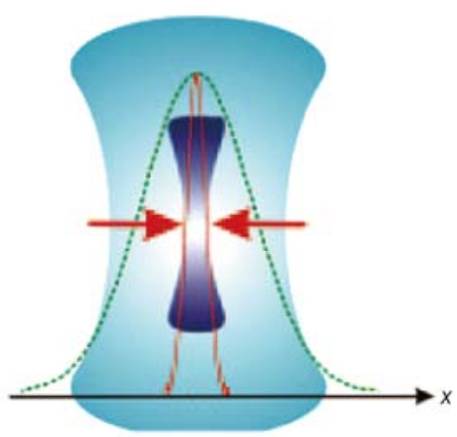

Figure 5 (Color online) The principle of reversible saturable optical fluorescence transitions [34]. Image courtesy of National Academy of Sciences.

can accurately analyze the performance of this optical system. However, with increasing side lobe size, OTF is better than PSF for evaluating the real resolving power of this system. In this section, we will explain how structured illumination methods break the diffraction limit in the frequency domain.

Structure illumination microscopy (SIM) is based on Moiré fringes detection. As can be seen in Figure 6(a), it is much easier to see the distortion of the fine structure by overlapping another known frequency. Similarly, with structured illumination, it is possible to obtain the highfrequency component of a sample from the illumination frequency and the easily measured Moiré fringe frequency alternations [36].

SIM is a method that can work beyond the diffraction limit (to reach $1 / 2$ diffraction limit), but is not unlimitedly in its ability to break the diffraction barrier. By incorporating the non-linear saturation effect into SIM, SSIM/SPEM has successfully achieved super-resolution and high quality images can be obtained by shifting the phase multiple times. By saturating illumination, higher frequencies (breaking the diffraction limit barrier) can be obtained. When changing the phase of the modulated light structure, the original frequency range will be extended to a larger range as shown on the right in Figure 6(c) [35].

Compared with other technologies mentioned above, the SSIM/SPEM developed by Gustafsson [35] used saturated structured illumination and has the advantage that no laser scanning is required. It can be treated as an opposite process to STED, although the SSIM/SPEM also uses the non-linear saturation effect $[53,62]$. The STED method makes a large number of fluorescent molecules stay in the $S_{0}$ state, while a few molecules are in $S_{1}$ and emit fluorescence. However, the SSIM/SPEM stimulates saturated fluorophores by applying striped array illumination as the excitation light, greatly confining molecules in $\mathrm{S}_{0}$ to form a dark region with steep edges surrounded by fluorescence in $S_{1}$ state. Only a small amount of non-fluorescent molecules exist among the excited fluorophores, and these small dark areas can be considered as the "complementary information" to the desired result [38]. Through computer decoding and inverse operations an original super-resolution image of samples can be obtained.

SSIM/SPEM can achieve faster imaging speeds but at the cost of relatively poor resolution because of the use of wide-field illumination, as opposed to several RESOLFTs that are based on confocal scanning technology. Currently the lateral resolution of SSIM/SPEM is $<50 \mathrm{~nm}$ [35]. This method can image an $8 \mu \mathrm{m} \times 8 \mu \mathrm{m}$ region in $0.9 \mathrm{~s}$ with a resolution of $100 \mathrm{~nm}$ [37], which is the fastest technology available in the field of super-resolution fluorescence microscopy for imaging large regions. Theoretically, as long as the stimulating light intensity increases, it can enhance the non-linear saturation effect to an infinite expansion of 


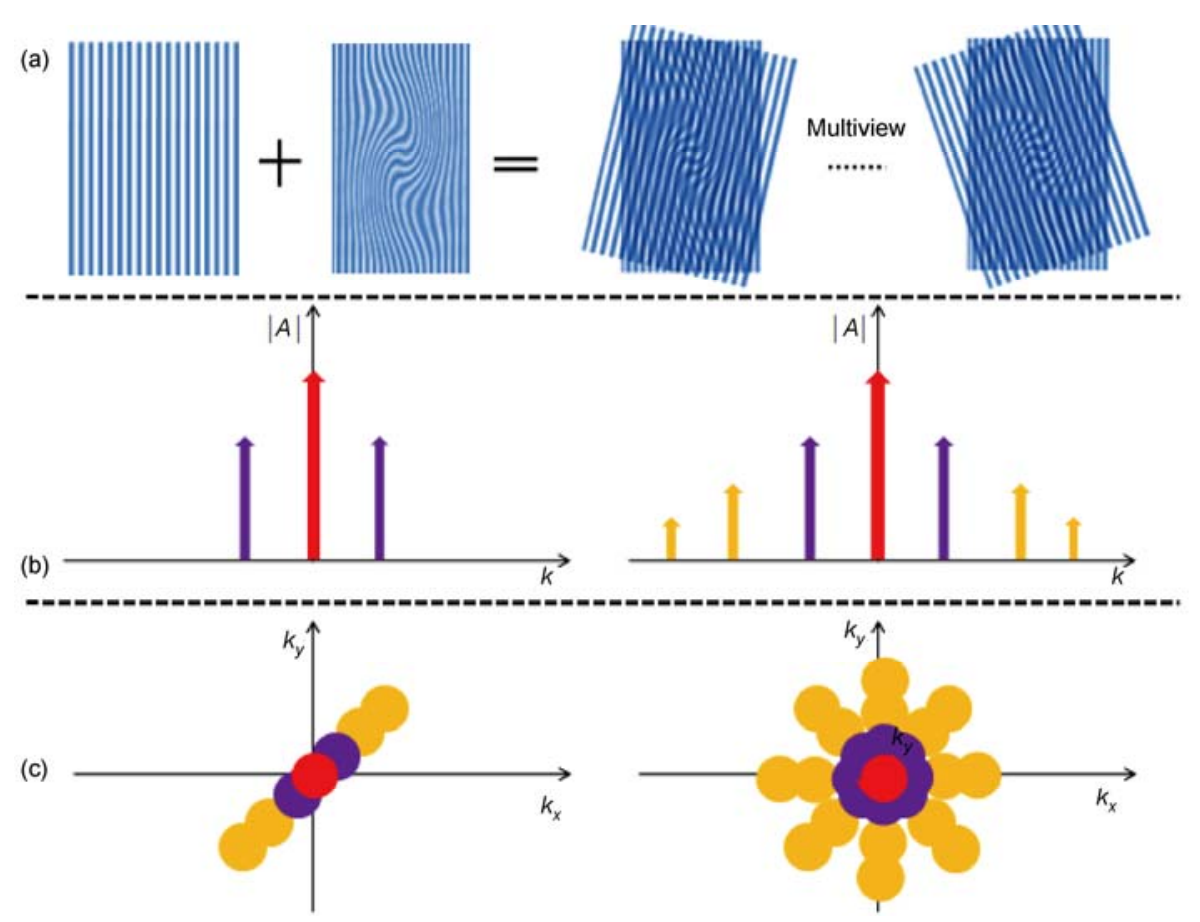

Figure 6 (Color online) (a) Illustration of the SIM in the spatial domain. The left schematic shows the modulated illumination structure, while the right schematic shows the sample with high-frequency. Distinct Moiré fringes appear as these are superposed. Rotating the illumination pattern can obtain the higher-order frequency information in multiple directions. (b) Illustration of SIM in the frequency domain. The central arrow indicate detected frequency taken from traditional optical microscopy; the two neighboring arrows represent additional information by SIM; other arrows are extra-frequency information obtained by SSIM/SPEM. With the increasing of frequency, the amplitude decreases so it is difficult to measure. (c) Phase analysis. The arrows in (b) are represented by colored circles and reveal the significance of the multi-view. The left panel shows detection fields under only one illumination angle, and the right shows expanded fields when phase shifting.

OTF. However, there still exists three issues: (1) The unlimited intensity of light can easily cause photo-bleaching and photo-damage same as seen with STED, GSD and other methods; (2) As shown in Figure 6(b), the amplitude of higher-order non-linear terms declines with the rise of the order, so not each term can be used to reveal details. When the signal to noise ratio is lower than 1, resolving power of the optical system is again limited; and (3) The same dilemma between resolution and imaging speed seriously restricts the development of SSIM/SPEM, in that the appearance of higher frequencies increases computational complexity and is more time-consuming.

\subsection{Stochastic excitation microscopy}

(i) Photo activated localization microscopy. In 2006, Betzig et al. [39], developed PALM technology, which is a randomly stimulating method to improve resolving power. The foundation of this method is that fluorescent molecules in one Airy disk can be activated one by one and then permanently bleached in the same order, and utilizes singlemolecular fluorescence microscopy to compute and map the precise position of each molecular center after several cycles, and then re-constitutes a complete image. The use of special fluorescent molecules is also the key to success in PALM. A special molecule, PA-GFP, is widely applied in
PALM because of its specific characteristics, such that only after activation with a $405 \mathrm{~nm}$ beam and subsequent $561 \mathrm{~nm}$ light will fluorescent emission occur [39]. Hess et al. [41] introduced fluorescence photoactivation localization microscopy (FPALM) technology in 2006, which is similar to PALM.

Specific procedure for FPALM is $[39,43]$ : a few proteins tagged by PA-GFP are activated with low intensity $\left(\mathrm{mW} / \mathrm{cm}^{2}\right) 405 \mathrm{~nm}$ laser pulse; then a $561 \mathrm{~nm}$ continuous wave stimulates molecules, although this results in a low amount of fluorescent emission because only a small number of elements were previously activated. By calculating and positioning the centers of these molecules their precise location can be obtained. When the $561 \mathrm{~nm}$ laser illuminates for a long time, the excited molecules will be bleached, and as a result they cannot participate in the following cycle, that is, the signal-to-noise ratio of the next process will not be influenced by the former steps. Subsequently, the accurate position of each molecule can be fitted according to real image results owning to the fact that it is usually a Gaussian distribution of fluorescent energy. One by one, by synthesizing all the former fluorescent images, the final result is achieved.

The advantage of this technique is, however, also its disadvantage: only a few molecules or even single elements participate in each cycle. Although discerning ability of this 
fluorescence nanoscopy technique improves in a great scale, imaging speed drops and the field of view is considerably reduced because of the transformation from highly-accurate positioning by the single-molecule imaging technique to subdiffraction microscopy. In Figure 7, the areas of $F$ and $F^{\prime}$ are $1 \mu \mathrm{m} \times 1 \mu \mathrm{m}$, and the others are $4 \mu \mathrm{m} \times 4 \mu \mathrm{m}$. Acquisition time is a consideration as the imaging speed is supposed to be $0.5-1 \mathrm{~s}$, so it will take $2-12 \mathrm{~h}$ to detect $10^{4}-10^{5}$ molecules [38]. Thus, this speed limitation further hinders the future developments using PALM in vivo.

On account of this drawback, two solutions were launched according to different research needs.

(1) Currently three methods are widely recognized in the pursuit of subdiffraction resolution.

(a) The PALM signal is relatively weak, so if total internal reflection fluorescence microscopy (TIRF) is equipped with the PALM system, the resolving power may be

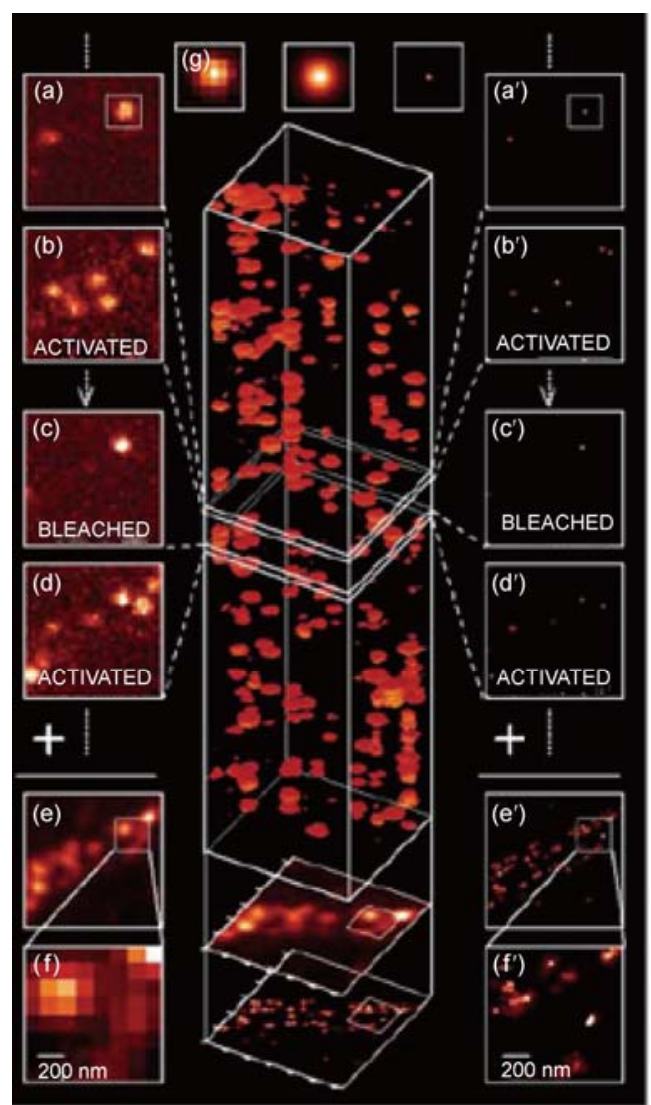

Figure 7 (Color online) The principle and procedure of fluorescence photoactivation localization microscopy. (a)-(c) After the PA-GFP fluorophore is attached to the target proteins, a $405 \mathrm{~nm}$ pulse activates only a few elements at one time and then $561 \mathrm{~nm}$ laser excites molecular emission until photo-bleaching occur. (d) The process shown (a)-(c) is repeated again and again until all the molecules are bleached and have lost the ability to be activated. (e), (f) The imaging results, which are limited by diffraction, will be superposed on a map. ( $\mathrm{g}$ ) Left side reveals the actual results of one molecule, the middle panel shows an ideal PSF of one spot, and the right panel shows the result of fitting the first two by standard deviation. $\left(a^{\prime}\right)-\left(f^{\prime}\right)$ correspond to the fitting results of each previous step [39]. Image courtesy of the American Association for the Advancement of Science. increased to the same order of magnitude as transmission electron microscopy (TEM). Furthermore, TIRF can reflect the contours of cells, while PALM can reveal their internal details. At present, outside mitochondrial membrane, the technology can achieve a lateral resolution of $20 \mathrm{~nm}$, decreasing the resolution up to 10 -fold of the conventional microscopy [39], realizing a transformation from highprecision positioning to super-resolution. Simple and cheap equipment are also merits.

(b) Recently, Shroff et al., put forward a two-color PALM system that utilizes two different types of fluorophores, Eos and Dronpa, to mark differentiated proteins. They used the reversible characteristics of Dronpa and the same beam to activate different molecules in order to identify spatial structures (Figure 8) [45].

(c) In 2010, Chu et al. [63], claimed that with absolute accuracy of $0.77 \mathrm{~nm}$ in a measurement of the separation between distinct colored fluorescent molecules at room temperature, the resolution of 1-2 $\mathrm{nm}$ can be achieved by this far-field fluorescence imaging mode.

(2) The pursuit of improving imaging speed has followed two methods $[38,42,44]$.

(a) Reducing the concentration of the fluorophore, but at the same time sacrificing discerning capability. This method causes a loss of the detailed information of a sample by cutting down the number of cycles.

(b) Increasing the excitation intensity at the expense of the lifetime of the fluorescent molecules. This approach induces a flicker effect, which, once it has occurred, not only introduces noise for the next cycle, but also increases the single imaging time.

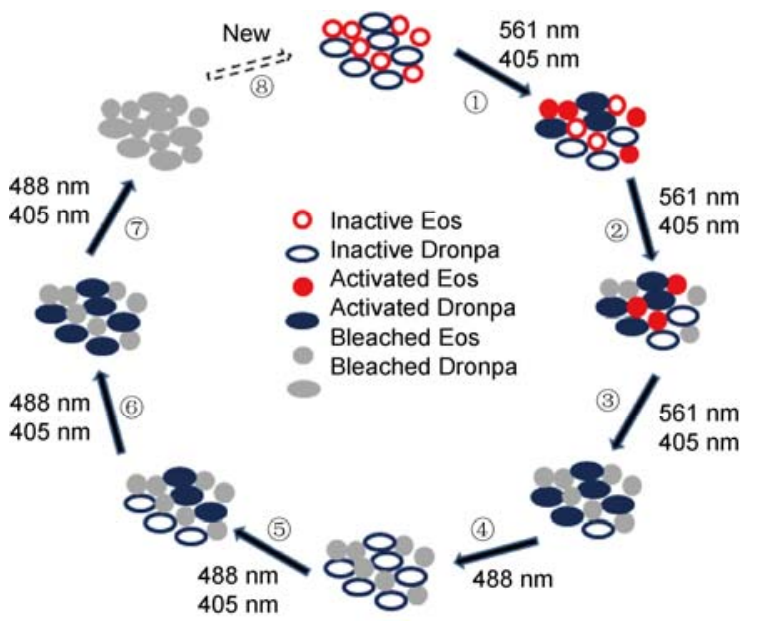

Figure 8 (Color online) The principle of two-color PALM. Nonactivated Eos (circles) and Dronpa (ovals) are fixed in the sample. The process in (1)-(3) is similar to PALM. The $405 \mathrm{~nm}$ beam activates a few Eos and Dronpa molecules; however, the $561 \mathrm{~nm}$ laser does not excite the Eos molecules to emit fluorescence until they are positioned and bleached. (4) The excited Dronpa is depleted to the original inactive state using the $488 \mathrm{~nm}$ beam. (5)-(7) The $488 \mathrm{~nm}$ beam stimulates Dronpa molecules to radiate, and then detection, location, and bleaching. (8) New cycle of next area begins. 
(ii) Stochastic optical reconstruction microscopy. STORM $[49,50]$ uses a technique in which Cy3 and Cy5 proteins cascade together as a source and can be repeatedly activated: illumination with different wavelengths makes Cy5 go through a process of "activation and excitation $\rightarrow$ emission $\rightarrow$ bleaching $\rightarrow$ recovery $\rightarrow$ activation" hundreds of times, until a permanent bleaching eventually occurs. Subdiffraction resolution is achieved after stochastically stimulating fluorescent molecules to locate the exact center of each available element.

The approach can be described as [50]: a $30 \mathrm{~W} / \mathrm{cm}^{2}$ red beam, whose wavelength is $633 \mathrm{~nm}$, illuminates the sample in order to force all the Cy5 to $S_{0}$; then a $1 \mathrm{~W} / \mathrm{cm}^{2} 532 \mathrm{~nm}$ green beam activates several $\mathrm{Cy} 3$ to pull $\mathrm{Cy} 5$ from a non-fluorescence state to $S_{1}$ in order to restore the capacity of fluorescence emission; at this time a $633 \mathrm{~nm}$ beam excites a few fluorescent molecules to make them release fluorescence and then go back to the non-fluorescent state again, in preparation for the next cycle. It should be emphasized that each Cy5 molecule can be activated several times in STORM until it is completely bleached, so one point of orientation is the result of multiple measurements due to multiple Cy5-Cy3 pair activation and measurement. It could be concluded that STORM contains several PALM processes, that is, in PALM one point is located only once. The accurate positioning depends on the number of photons emitted from a single molecule, so when the molecule experiences 20 or more cycles, the resolution is up to $20 \mathrm{~nm}$ after fitting [50]. From the perspective of statistics, STORM maintains higher positioning precision than PALM $[38,55]$.

(iii) Direct stochastic optical reconstruction microscopy. Direct stochastic optical reconstruction microscopy (dSTORM) switches and reads information stochastically like STORM, and the only difference is the direct application of the traditional Cy5 or Alexa647 fluorophore instead of $\mathrm{Cy} 3$ [51]. Hence, there is no need for specific binding two different kinds of fluorescent molecules to the proteins, so the complexity of the experimental equipment is reduced.

For $647 \mathrm{~nm}$ stimulating beam power, it is proportional to the probability of back to the non-fluorescent state of $\mathrm{Cy} 5$, while the power of $514 \mathrm{~nm}$ activation is the same as probability of up to the fluorescent state [51]. Although the power of dSTORM is 200 times greater than that of STORM, under the experimental conditions the amplitude is still permitted. Thus, by adjusting the laser power, the method of switching molecules to transit between two states on purpose with stochastically recording the exact position centers become true. Figure 9 contrasts the results between dSTORM and traditional microscopy.

It has been reported that the lateral resolution of dSTORM reached $20-30 \mathrm{~nm}$ and an axial resolution of 50-60 nm [51]. Currently, the fluorophore Cy5, Cy7, Alexa647, and Alexa680 can be explored using dSTORM. Furthermore, it greatly enhances the super-resolution microscopy because of its brilliant ability to discern complex

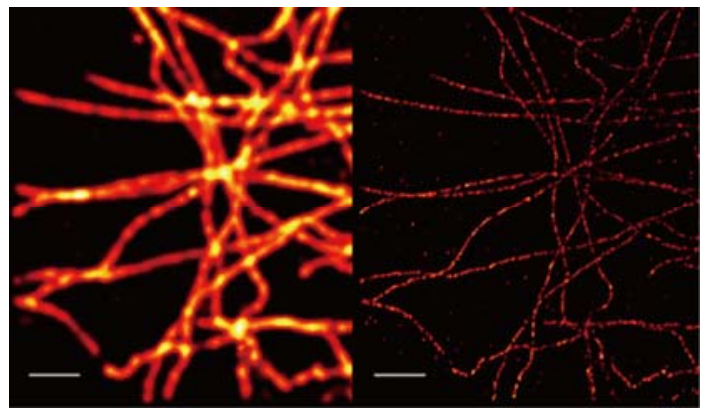

Figure 9 (Color online) A comparison between dSTORM and traditional microscopy. The left side shows the result of traditional fluorescence microscopy; the right side shows image by dSTORM. Scale bar: $2 \mu \mathrm{m}$. (http://www.zeiss.com/C12567BE0045ACF1/ContentsFrame/7EFC4393ABB349F9C125758D00210C72).

spatial structures and the application of single molecules by multi-color imaging similar to that with two-color PALM [16,38,51].

This series of stochastic mode microscopy techniques, such as PALM and STORM, characterized by low photodamage with a low laser intensity and power, need to be improved further in order to be useful for in vivo imaging due to increased imaging time after many cycles concentrating on a small field of view. At the same time, there has been an obvious increase in the integration of different methods that are gradually becoming available with regard to two-color PALM and dSTORM. As indicated above, a multi-spectrum method is not only easier but faster than sequent or stochastic approaches as long as it has the ability to work beyond the diffraction limit. Therefore, with the robust development of PALM and STORM and the exceptional confinement of PSF, integrating methods such as multi-spectrum technology and GSDIM approaches can expect a prominent future in the field.

(iv) Ground state depletion-individual molecule return microscopy. In 2008, starting with GSDIM technology, super-resolution fluorescence microscopy raised the curtain on integration between ground state depletion and the stochastic switching mode. GSDIM combines and makes full use of the advantages of both the GSD method and singlemolecule fluorescence imaging approaches to achieve subdiffraction resolution. Rh6G and Atto532 fluorophores are employed in GSDIM [52].

The common ground among PALM, STORM and GSDIM is that all rely on the stochastic mode, but the former two methods need to stimulate the fluorophores to emit fluorescent light by excitation, while the GSDIM depletes unwanted elements, similar to the GSD method, to realize spontaneous fluorescent emission. That raising molecules from $S_{0}$ to the metastable or other triplet states makes them locate between two states so their emission ability is loss. Thus, it is not necessary to alternately stimulate with multiple wavelengths. When most molecules are forced into the short lifetime $S_{0}$ state, the center of fluorophore can be located according to the spontaneously released photons from 
the remaining molecules [16,53].

The difference between GSDIM and GSD lies in the fact that the former limits the number of photons emitted and accurately locates the position of the center of the fluorophore by single molecule fluorescence imaging rather than by confining the PSF by stimulation. During experiments, each fluorescent molecule need only participate in one cycle and then returns back to the $\mathrm{S}_{0}$ state, unlike GSD, which pumps repeatedly, thus greatly reducing the possibility of photo-damage because of reduced cycle-times. In Figure 10, dual-color GSDIM further combines the merits of different modes, by applying just one group of continuous wave lasers to act as not only excitation but also depletion beams, thus greatly simplifying equipment; lateral resolution of < $30 \mathrm{~nm}$ can be achieved [52].

(v) Point accumulation for imaging in nanoscale topography. Sharonov and Hochstrasser [57] used fluorescent molecules diffusing in solution as a probe to achieve a subdiffraction resolution by continuous collisions to the sample. The principle of the method suggests that molecules are attached to the sample after collisions and emit fluorescence, while the others stay in solution and have no ability to radiate. Next, according to the location of the fluorescence, the precise position of each fluorophore is fitted by a Gaussian function to obtain the absolute center. Notably, the fluorescence is still limited by diffraction until the fixed molecules detach from the sample, at which point light will be extinguished. It is not difficult to control the rate of molecular collisions as the rate is proportional to the concentration of probe. If the measured sample is sensitive to the chemical structure, distinguishing different molecular positions will be easy in the resulting images [55,57,58].

During the process of PAINT, it is necessary to ensure that less than one molecular probe/ $\mu \mathrm{m}^{2}$ is captured in each sampling period. To meet this need, the time interval between successive frames should be longer than the lifetime
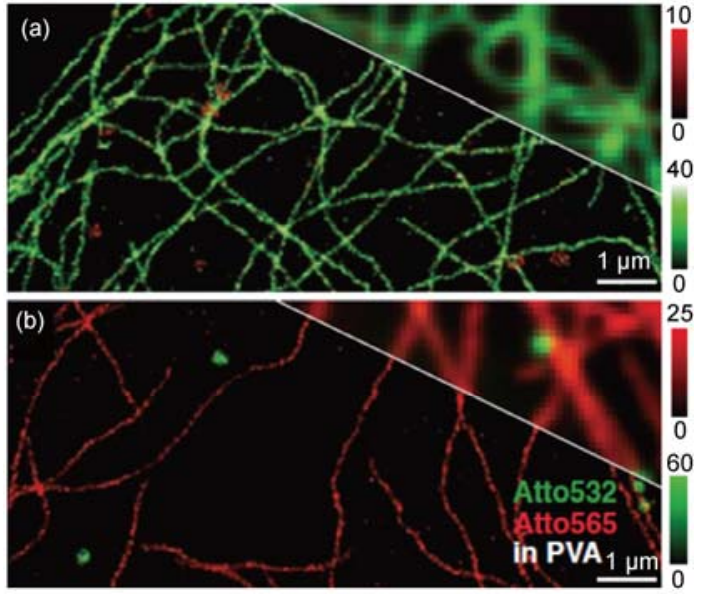

Figure 10 (Color online) Imaging results of GSDIM using Atto532 and Atto565. (a) Microtubules; (b) peroxysomes of PtK2-cells embedded in PVA. The right corners are the results using epifluorescence microscopy [52]. Image courtesy of Nature Publishing Group. of each molecule, but the time of a single frame should be shorter than the interval of continuous collisions. This guarantees that one molecule is recorded only once during two frames to obtain high precise positioning. The rate of photo-bleaching is closely related to the intensity, photochemical and photo-physical attributes of the molecular probe, which determines imaging speed [56].

Currently, PAINT can discern inter-object distances of $25 \mathrm{~nm}$ (Figure 11) [57], and can acquire fast images in $\mu \mathrm{m}$ regions in seconds. The major differences between PAINT and the other technologies introduced above are [55,56]:

(1) There is no need to mark the sample by a fluorophore in advance;

(2) All the fluorescent probes are suitable for PAINT, so choosing suitable fluorophore sensitivity is essential;

(3) The number of photons released by molecules is flexible because a large amount of the fluorophore is involved in the process;

(4) A liquid interface is needed.

\subsection{Comparison among sequential, structured, and stochastic switching modes}

A comparison among the sequential switching mode, structured switching mode and the stochastic switching mode is shown in Figure 12. The names of the approaches are shown in the first column; in the second column STED, GSD, SSIM/SPEM, PALM, STORM and GSDIM are contrasted according to the energy levels of the molecules [53]. The third column points out the relationship between intensity and the number of molecules in the excited state in the first two switching mode, and characteristics of the stochastic switching mode. The right column illustrates that fundamental difference in principles of two modes, that is, the sequential and structured switching modes focuses on the ensemble, while the other mode aims at single molecules.

\section{Super-resolution fluorescence microscopy along the $Z$-axis}

In traditional optical microscopy, or even confocal micros-
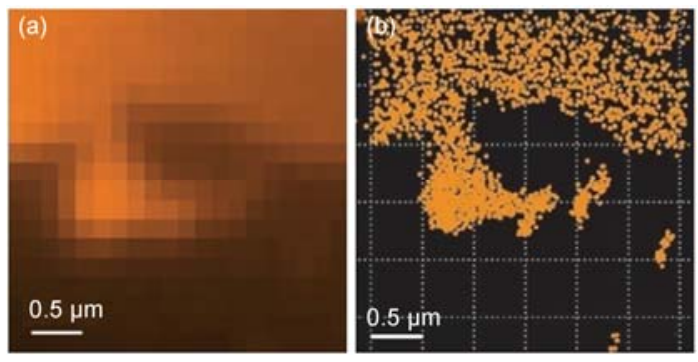

Figure 11 (Color online) Comparison of a supported bilayer on glass, probed by Nile red. (a) Image of conventional fluorescence microscopy; (b) image using PAINT [57]. Image courtesy of National Academy of Sciences. 




Figure 12 (Color online) A comparison among the sequential switching mode, structured switching mode and the stochastic switching mode.

copy, the axial resolution is three or four times lower than the lateral resolution, and has largely hindered the development of microscopy. According to the Abbe and Rayleigh criteria, by increasing the N.A. of the system, resolving power can be improved to a certain degree, but unfortunately the semi-aperture angle $\alpha$ can, at most, reach $74^{\circ}$ [64], significantly departing from an ideal of $90^{\circ}$. Therefore, the resolution is always limited by diffraction. It should be emphasized that, axial resolution is inversely proportional to the square of N.A., so the demands of the increasing semi-aperture angle, $\alpha$, is of prime importance. From the perspective of physical principles, the disparity between axial resolution and lateral resolution is readily explained. The beam passing through the objective symmetrically focuses on the $X Y$-plane, but along the $Z$ axis it lacks symmetry because only one lens effects the focusing. At the same time, due to the existence of optical aberrations, the focal spot is stretched leading to a decline in axial resolution [53]. Recently, two types of technology can enhance axial resolution, but they fail to break the diffraction limit because the resolution is still relative to wavelength. Generally speaking, these are image incoherent interference illumination image interference microscopy $\left(\mathrm{I}^{5} \mathrm{M}\right)$ and $4 \mathrm{Pi}$ confocal microscopy, which enhances the $\alpha$ angle, and light sheetbased fluorescence microscopy (LSFM), including digital scanned laser light sheet fluorescence microscopy (DSLM), selective plane illumination microscopy (SPIM) and so on $[65,66]$, which stimulate the sample by planar illumination. The latter transforms the measurement of axial resolution to lateral one, so it can achieve the same order of lateral resolution.

\subsection{The opposing lenses method}

The threshold of opposing lenses can be used to expand the aperture-angle of an objective lens, so that the wave-front is more spherical in wide-field illumination and confocal microscopy. Owing to the fact that the aperture-angle is limited for one lens, another objective lens is placed in the opposite position, to decrease the focus by interference of the two beams. This approach has been applied in $\mathrm{I}^{5} \mathrm{M}$ to enhance the axial resolution of wide-field illumination, and 4Pi microscopy, and can also be adapted to confocal laser scanning microscopy $[16,38,53]$.

(i) Incoherent interference illumination image interference microscopy. $\quad I^{5} \mathrm{M}$ is based on image interference microscopy $\left(\mathrm{I}^{2} \mathrm{M}\right)$ and incoherent interference illumination microscopy $\left(\mathrm{I}^{3} \mathrm{M}\right)$. The basic principle of $\mathrm{I}^{2} \mathrm{M}$ is shown in Figure 13(a), and features fluorescence interference. The sample is set between the opposing lenses so that laser beams from two directions are focused on the same area, and then by adjusting them to the same optical distance, the CCD detects the common information through fluorescence interference. In $\mathrm{I}^{2} \mathrm{M}$, the two fluorescence beams are transmitted through two objective lenses, respectively, while only the stimulation beam propagates through one lens; $I^{3} \mathrm{M}$ uses interference of the illumination beams, to obtain high-frequency interference signals from spatially incoherent light. After combination of both $\mathrm{I}^{2} \mathrm{M}$ and $\mathrm{I}^{3} \mathrm{M}$, resolution can be up to $70 \mathrm{~nm}$, which improved axial resolution by up to 7-fold and was 2 and 3.5 times greater respectively than that of $I^{2} \mathrm{M}$ or $\mathrm{I}^{3} \mathrm{M}$ alone [67]; this ability of $\mathrm{I}^{5} \mathrm{M}$ is shown in Figure 13(b). The image information arises not only from fluorescence interference but also stimulation.

Although its axial resolution is higher than that of conventional microscopy and the imaging speed is faster than confocal microscopy, $\mathrm{I}^{5} \mathrm{M}$, based on wide-field illumination microscopy still has the drawback of involvement from non-focal information. It brings about the expansion of the side lobes of the PSF and reduces high-frequency information of the OTF, which raises difficulties for de-convolution. Moreover, a majority of $\mathrm{I}^{5} \mathrm{M}$ techniques employ oil immersion objectives, and therefore are not suitable for observing the sample in vivo $[38,67]$.

(ii) 4Pi confocal microscopy. 4Pi confocal microscopy, like $\mathrm{I}^{5} \mathrm{M}$, places two opposing objective lenses on either side of the sample. The addition of a digital aperture to control the focus of the illumination on the same area can, theoreti- 


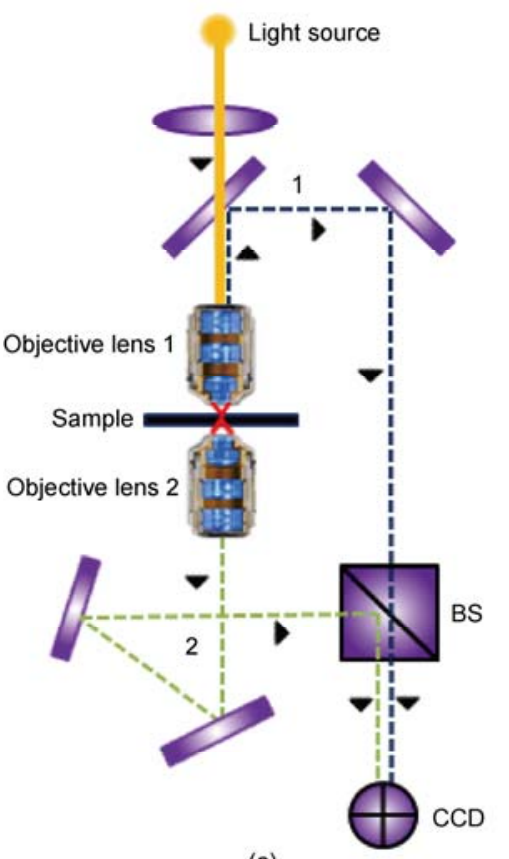

(a)

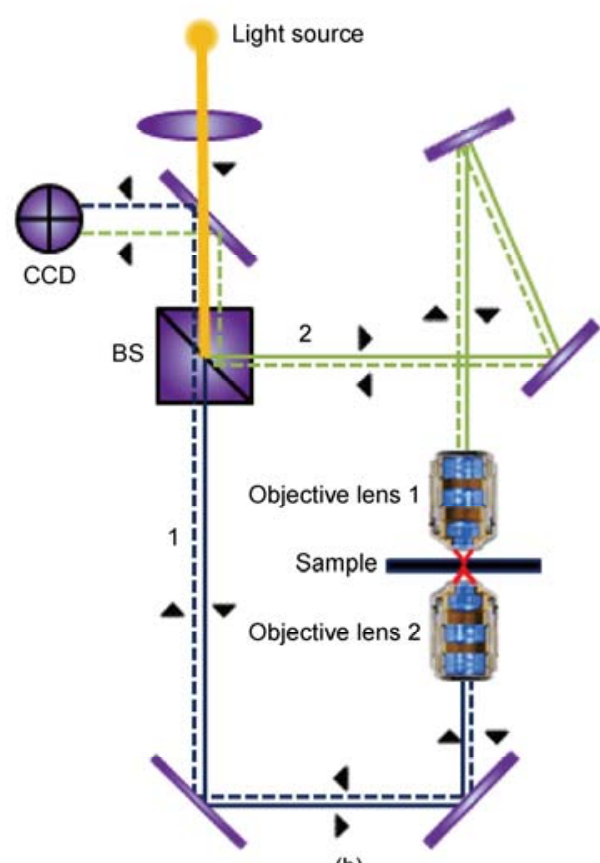

(b)

Figure 13 (Color online) $\mathrm{I}^{2} \mathrm{M}$ (a) and $\mathrm{I}^{5} \mathrm{M}$ (b). Arrowheads represent the direction of light propagation.

cally, sample information from $4 \pi$ fields, hence the nomenclature of 4Pi confocal microscopy (Figure 14) [68-70]. The difference between the $4 \mathrm{Pi}$ and $\mathrm{I}^{5} \mathrm{M}$ methods is the stimulation approach of $4 \mathrm{Pi}$, which adopts a scanning spot by spot regime instead of wide-field excitation. Although 4Pi confocal microscopy is slower than $I^{5} \mathrm{M}$, the advantages are high-quality OTF, outstanding transmission of highfrequency, and no forbidden-bands in the frequency field. According to different interference types, the 4Pi method is divided to three modes: 4Pi-A, $-\mathrm{B}$ and $-\mathrm{C}$. Among them, 4Pi-A depends on stimulation beams interference, while $4 \mathrm{Pi}-\mathrm{B}$ is similar to $\mathrm{I}^{2} \mathrm{M}$, and $4 \mathrm{Pi}-\mathrm{C}$ combines the first two techniques and has the highest resolution [64,71].

In order to effectively suppress the side lobes of OTF, 4Pi confocal microscopy was first combined with twophoton microscopy, which reduced non-focal vertical intensity. The OTF of 4Pi-C is close to isotropy as the side lobes are only $25 \%$ of main body, which is easy to de-convolute during the imaging process $[38,72]$. Meanwhile, with reduction of the side lobes, the requirement for N.A. decreases and it is possible to use water immersion lens to observe living cells in situ. After dual-color two-photon microscopy is added, axial and lateral resolution reaches 57 and $97 \mathrm{~nm}$, respectively [73]. However, mismatching of the refractive index caused by dispersion in two-photon process increases the complexity of the experiment.

A breakthrough in the field of image speed enhancement has been made possible through the use of multifocal multiphoton 4Pi microscopy (MMM-4Pi). With a micro-lens array, a number of stimulation beams can be divided into more beams that simultaneously focus on their own focal points. Therefore, imaging speed is greatly enhanced. According to reports, this method can detect a $20 \mu \mathrm{m} \times 20 \mu \mathrm{m} \times$ $5 \mu \mathrm{m}$ area in $100 \mathrm{~s}$ with a $100 \mathrm{~nm}$ resolution [15,56]. However, at present the complicated and costly equipment constrain the development of MMM-4Pi.

Comparison of the contrast results shown in Figure 15, makes it clear that these two novel microscopy methods, 4Pi and $\mathrm{I}^{5} \mathrm{M}$, not only meet the needs of traditional microscopy standards, but also, to some degree, extend the frequency range and acquire more high-frequency details. Furthermore, in the frequency domain, the OTF of 4Pi approach is continuous, while the one of $\mathrm{I}^{5} \mathrm{M}$ is discrete. The discrete region, which is the black areas among purple patterns, is shown in Figure 15(c). Comparison between Figure 15(a) and (b), shows that the frequency range of $4 \mathrm{Pi}-\mathrm{C}$ confocal micros-



Figure 14 (Color online) The principle of 4Pi-C microscopy. Arrowheads represent the direction of light propagation. 

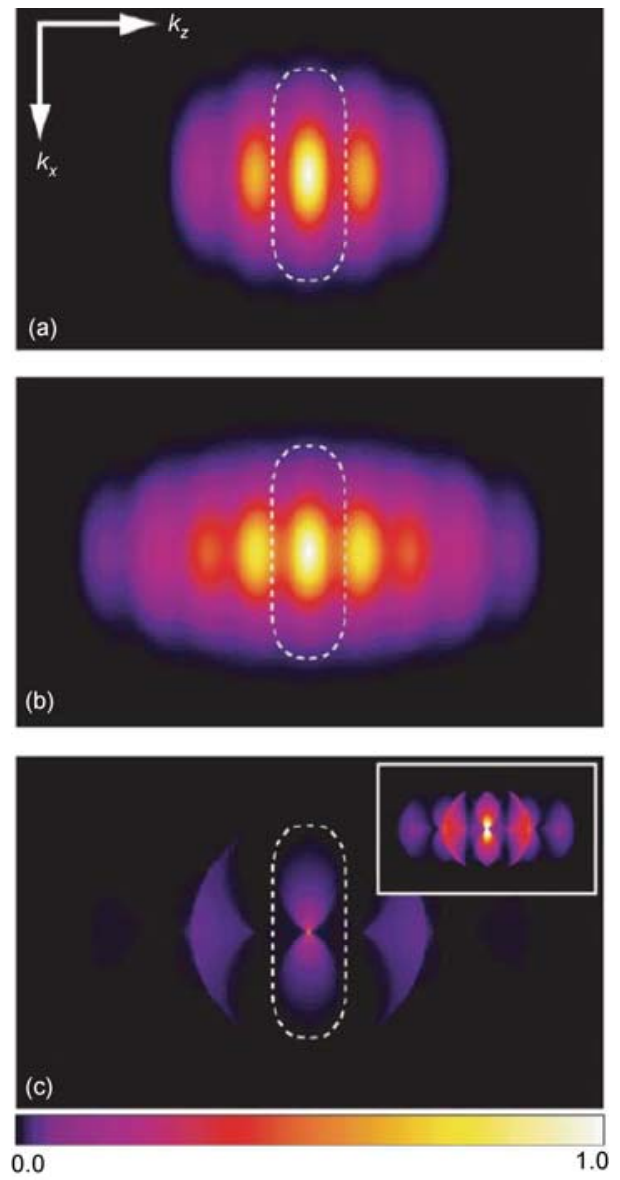

Figure 15 (Color online) Comparison of OTFs using different microscopy methods. (a) represents the OTF using 4Pi-A confocal microscopy with a two-photon microscope; (b) shows the OTF using 4Pi-C confocal microscopy with a two-photon microscope; (c) illustrates the OTF using $I^{5} \mathrm{M}$. The intensity is pseudo-colored. In the white dashed region, the OTF of confocal microscopy indicates the observed frequency field seen under the same condition using confocal microscopy [71]. Image courtesy of Elsevier.

copy is clearly broader than that of $4 \mathrm{Pi}-\mathrm{A}$, with the result that $4 \mathrm{Pi}-\mathrm{C}$ has higher resolution and better image quality [38].

Compared with conventional microscopy, which employs only one objective lens, $\mathrm{I}^{5} \mathrm{M}$ and $4 \mathrm{Pi}$ microscopy are more vulnerable to influences caused by a mismatch or uneven distribution of refractive indexes that can easily cause focal plane deviations and distortions. Therefore, the method requires samples with good transparency and thickness ranging from a few to tens of microns.

\subsection{Light sheet-based fluorescence microscopy}

Although all of the previous methods reduce the complexity of equipment and the experimental approaches to the optics, using the same path for the illumination beam and fluorescence leads to intensive photo-bleaching and photo-toxicity (Figure 16(a)). On account of this disadvantage, LSFM splits the paths so that the illumination path is perpendicular to the detection direction. Fluorescence emits only from the excited focal plane. Along the direction of detection photo-bleaching and photo-toxicity are dramatically decreased, because there a few fluorescent molecules are excited in the micron thickness. LSFM includes DSLM, SPIM and ultramicroscopy techniques, all of which have the same principles, whereas the main differences concentrate on the configuration of pinholes, lenses, mirrors, laser sources and other devices [65,66].

As a representative of LSFM, digital scanned laser light sheet fluorescence microscopy (DSLM) integrates various methods, and compared with conventional microscopy it has several advantages [65].

(1) Signal efficiency: DSLM can illuminate the desired sample area, to large extent increasing signal efficiency.

(2) Energy efficiency: in the absence of a pinhole, the loss of energy efficiency of the illumination beam is not more than $5 \%$ after transmissionion through the lenses and mirrors to scan a plane of sample. Furthermore, the light sheet reduces the energy required for stimulation by about $10^{3}-10^{6}$ times over conventional excitation, thus greatly decreasing photo-bleaching and photo-toxicity.

(3) Resolution: the axial resolution of DSLM is improved through the light sheet imaging.

(4) Imaging speed: after applying a CCD with a large dynamic range, the imaging speed of DSLM is up to 63 $\mathrm{Mpixel} / \mathrm{s}$, far more than the 2-6 Mpixel/s of confocal microscopy and $1 \mathrm{Mpixel} / \mathrm{s}$ of two-photon microscopy.

(5) Signal-to-noise ratio: The signal-to-noise ratio of DSLM is about for 1000: 1, allowing for higher qualitative image, while that of confocal microscopy is $60: 1$ and twophoton microscopy only $10: 1$.

\section{Three-dimensional super-resolution fluores- cence microscopy}

\subsection{Three-dimensional STED imaging}

(i) Isotropic STED. Isotropic STED (isoSTED) [16,74], also known as the STED-4Pi technique, is a combination of STED and 4Pi confocal microscopy, and can reach the same resolution in both lateral and axial imaging. As a representative method of improving microscopic resolution, STED and 4Pi were both proposed in the 1990s by Schmidt and colleagues [74]. At present, isoSTED has already improved the resolution in three-dimensional space by up to $30 \mathrm{~nm}$.

Based on STED, isoSTED depletes fluorescence in the $X Y$ plane and along the $Z$ axis using two STED beams $\left(\mathrm{STED}_{x y}, \mathrm{STED}_{z}\right)$, and adapts $4 \mathrm{Pi}$ confocal microscopy to effectively improve resolution by interference imaging (Figure 17). By controlling the polarizing directions with the rotation of two half-wave plates, the amplitude of the two polarizations can be matched when they are interfering. 

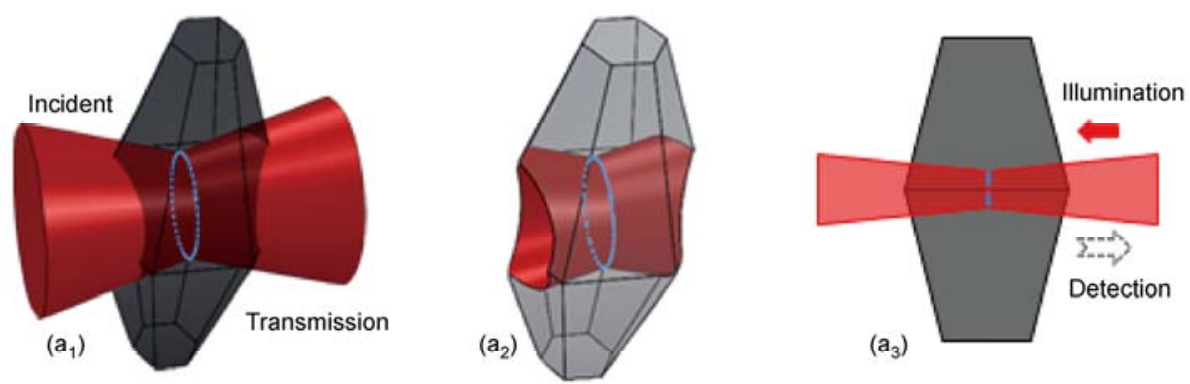

(a) Conventional \& confocal microscopy
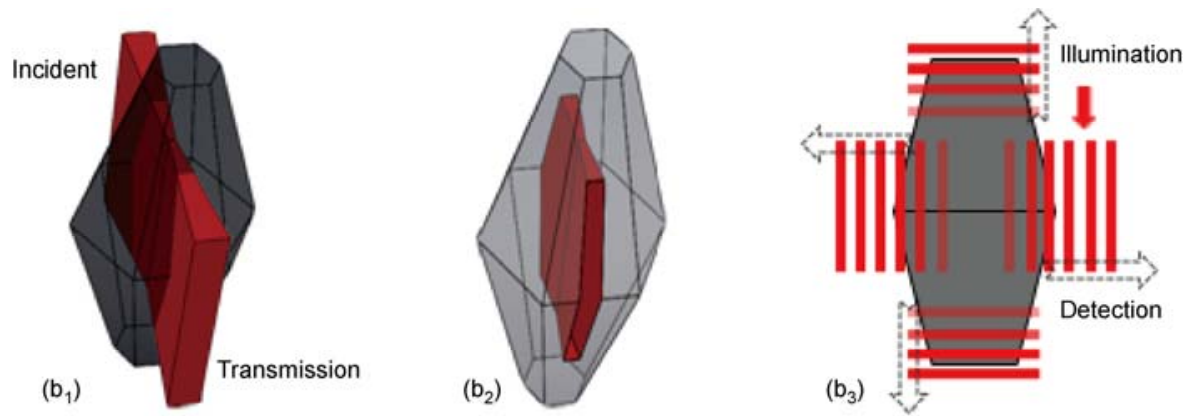

(b) Light sheet microscopy

Figure 16 (Color online) Contrasts between conventional microscopy and LSFM. Black or grey objects illustrate the sample and red are the areas illumination/excitation. $\left(a_{1}\right)$ In conventional microscopy, the illumination beam and fluorescence detection are in the same direction; the dash circle represents the focal plane. $\left(a_{2}\right)$. Although only focal plane signals are valuable, the samples are fully stimulated, so photo-damage occurs over a large area. ( $a_{3}$ ) Illumination and fluorescence detection are in the same path in conventional methods. $\left(b_{1}\right)$ The principle of LSFM. $\left(b_{2}\right)$ LSFM only illuminates the detected plane so that photo-damage is constrained to a small volume. $\left(\mathrm{b}_{3}\right)$ Directions of stimulation and detection are orthogonal. Multi-view can image large objects and collect images at different orientations for 3-D reconstructions.

(a)
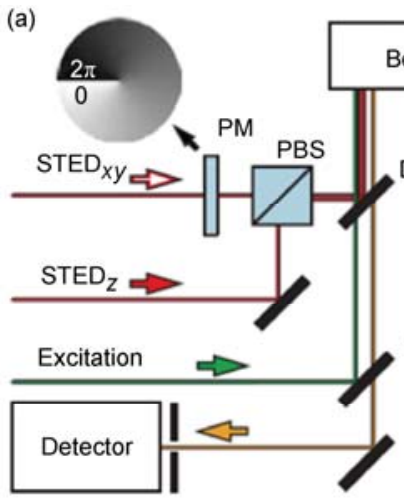

(b)

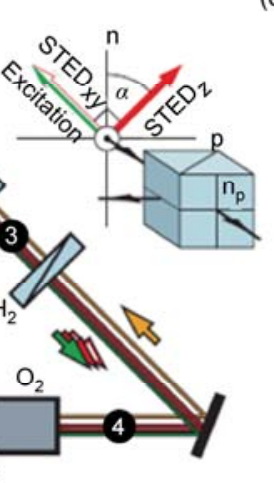

(c)
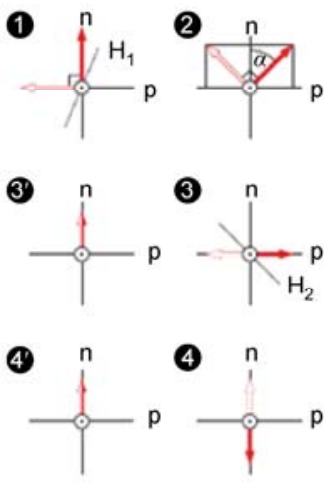

Figure 17 (Color online) The principle of isoSTED. (a) Excitation and STED beams $\left(\mathrm{STED}_{x y}, \mathrm{STED}_{z}\right.$ ) are separated after passing through a dichroic mirror (DCSP), into two opposing lenses $\mathrm{O}_{1}$ and $\mathrm{O}_{2}$, to reach the sample, $\mathrm{S}$, and come back to the detector via the same way after transmitting through the DCSP and the other dichroic mirror, DCLP. PM is a circular phase ramp, whose function is in charge of the optical density of STED $x y$; PBS is a polarizing beam-splitter, whose function is to separate the propagation of different polarized angles; $\mathrm{H}_{1}, \mathrm{H}_{2}$ are half-wave retarder plates, whose function are to modulate polarization states of $\mathrm{STED}_{x y}$ and $\mathrm{STED}_{z}$; MP is a piezo-driven mirror, which controls the difference in path-length between both cavity arms and the 4Pi phases of all beams; PP is a pivot plane that is conjugated to the entrance pupils of the objective lenses; dark circles indicate the polarization state at that


ized orthogonal to each other. (c) The polarization conditions of different paths, note, the relative phase differs by $\pi$ between 4 and 4 ' [74]. Image courtesy of Nature Publishing Group.

Then by guiding the STED beam on the $X Y$ plane $\left(\mathrm{STED}_{x y}\right)$ and the STED beam along the $Z$ axis $\left(\mathrm{STED}_{z}\right)$ one can obtain a compressed, uniform PSF on the focal plane. In addition, the polarization control can be replaced by employing different wavelength light.

\subsection{Three-dimensional STORM/PALM imaging technology}

(i) Imaging by cylindrical lenses. STORM and PALM are similar in principle and both, at first, did not have the ability to resolve the axial plane. Based on astigmatic principles, 
fine images along the $Z$-axis became a reality after assembling a cylindrical lens along the propagation path of the detection beam. When the detected fluorescent source is located on the focal plane, the spot on the CCD is a circle; yet when the source moves away from the focus, the spot on the CCD resembles an oval shape; whether the molecule of interest is above or beneath the focal plane can be judged according to the direction of the long axis of the ellipse. The lateral resolution of this approach can reach 20-30 nm, with an axial resolution of 50-60 nm [49]. Because of its simple experimental set-up and easy data analysis, cylindrical lens 3-D imaging technology has become widely recognized. However, two factors still influence measuring errors. Firstly, the accuracy of the cylindrical lens is limited penetration depths of about $600 \mathrm{~nm}$; secondly, as depth increases there is an increase in the extent of optical aberrations along the $Z$-axis and a steady deterioration of the image [43].

(ii) Double-helix point spread function. Pavani et al. proposed that a double-helix point spread function (DH-PSF) could obtain a subdiffraction resolution by modifying traditional PSF, similar to the methods proposed for STED and SSIM/SPEM. This change enhanced axial resolution to 10-20 nm within a $2 \mu \mathrm{m}$ imaging depth (Figure 18). The key point was to gain a DH-PSF by a special spatial light modulator (SLM). By judging the angle between links that are formed by two branches and the horizontal direction, it is possible to determine the position of a fluorophore along the $Z$-axis. The method can be adapted to single molecular imaging technologies. In order to resolve the bottleneck of low efficiency in photon collection and the difficulties in producing a SLM, we can add more phase modulators and closed-loop correction devices to enhance both the signalto-noise ratio and resolution [43,75].

(iii) Double PALM. Double-PALM simultaneously sends two different focal data sets to a CCD in order to extract information for three-dimensional image reconstruction. Currently, the lateral resolution has reached $30 \mathrm{~nm}$, axial resolution $75 \mathrm{~nm}$ and an imaging depth of about $1 \mu \mathrm{m}$ [43]. The illumination is divided into two beams by a 50:50 splitter and one of beam transits directly through the splitter, while the other is first reflected by the splitter and then reflected by a mirror. Because the optical paths are different the two beams contain information from different planes and both will image on the CCD. Therefore, the positions of molecules on the $Z$-axis can be detected. Compared with other methods, double-PALM has simpler instrumentation; however, the penetration and resolution need to be improved.

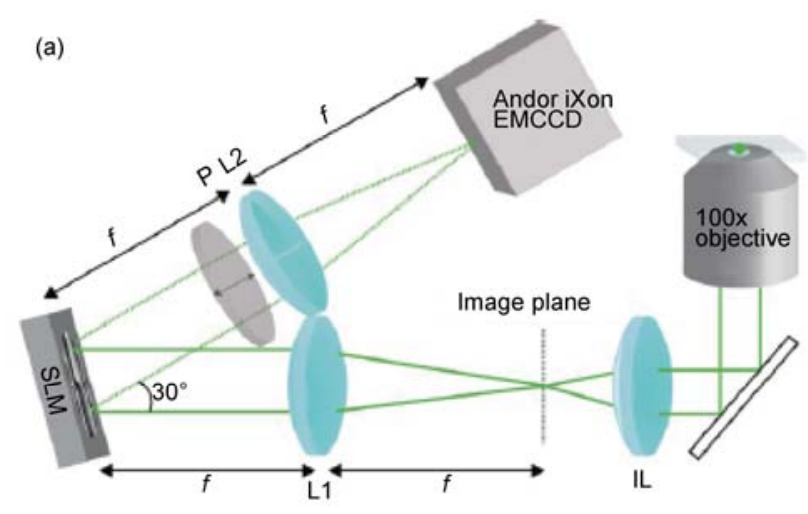

(c)

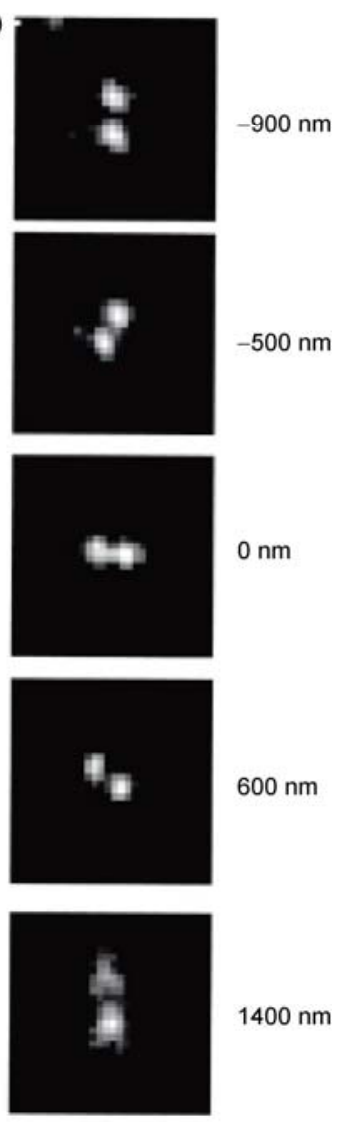

(b)



Figure 18 (Color online) The principle of DH-PSF. (a) Schematic of the device; (b) the relationship between the angel of links that connect each side of the leaves to the horizontal direction vs. the position of the light source on the Z-axis; (c) the position of the light source on the Z-axis at different angles [75]. Image courtesy of National Academy of Sciences. 
(iv) Interferometric PALM. Hess and LippincottSchwartz introduced the interferometric PALM (iPALM) method in which two opposing lenses are used in combination with PALM. iPALM became one of the leaders among the PALM series due to its $9.8 \mathrm{~nm}$ axial and $20 \mathrm{~nm}$ lateral resolution (Figure 19) [76]. iPALM uses fluorescence interference technology and simultaneous multi-phase measurement techniques to overcome the instability of fluorescence intensity and short lifetime of molecules, and has achieved remarkable results.

With the application of a multi-pass beam splitter, the multi-phase synchronous interference can quickly extract information from the sample as shown in Figure 19(a). When the light source is $\delta$ away from the focal plane, two different optical paths and phase differences are naturally produced. After the intervention of beam splitter, the phase difference modulates these three signal magnitudes on the CCDs. Therefore, the position of a molecule in $Z$-axis can be determined. The high precision positioning of PALM in a plane makes the iPALM three-dimensional detection a reality.

\subsection{Three-dimensional SIM imaging}

(i) 3-D SIM. By adding another interference beam into the optical path to extract Z-axis information, 3-D SIM was able to realize the goal of 3-D imaging, and achieved $100 \mathrm{~nm}$ lateral and $300 \mathrm{~nm}$ axial resolutions [77]. 3-D SIM has an advantages with respect to imaging speed and there is no need for a special fluorescent label (which makes the application convenient), although if no saturated nonlinearity is applied. Its three-dimensional resolution is still much lower than that of other super-resolution microscopy methods [43].

(ii) $I^{5} S . \quad I^{5} S[16,78]$, based on the SIM planar imaging method, is a new three-dimensional super-resolution fluorescence microscopy that incorporates the axial resolution advantages of $I^{5} \mathrm{M}$. It is natural to associate isoSTED with $I^{5}$ S. SIM has several characteristics in common with STED in the $X Y$ plane, the difference being that the former is a wide-field illumination microscopy mode, while the latter is a confocal laser-scanning mode. Therefore, it became necessary to combine two wide-field modes in order to create a new technique such as isoSTED. Shao et al. [78] recently reported that the resolution of $\mathrm{I}^{5} \mathrm{~S}$ in three dimensions has reached $100 \mathrm{~nm}$.

Similar to the $\mathrm{I}^{5} \mathrm{M}$ system shown in Figure 13(b), the whole device inserts an optical grating in addition to controlling the direction and phase structure of the illumination. Generally speaking, the excitation beams diffract after the optical grating and produce structured pattern, and some of beams practically stimulate the sample through a dispersion prism, the others excite the sample after reflection from the beam splitter. The fluorescence signal returns along the former optical path after excitation, as is received by the CCD after passing a dichroic mirror. Two of the four reflectors are movable in order to eliminate optical path differences. For the moment, the $100 \mathrm{~nm}$ spatial resolution

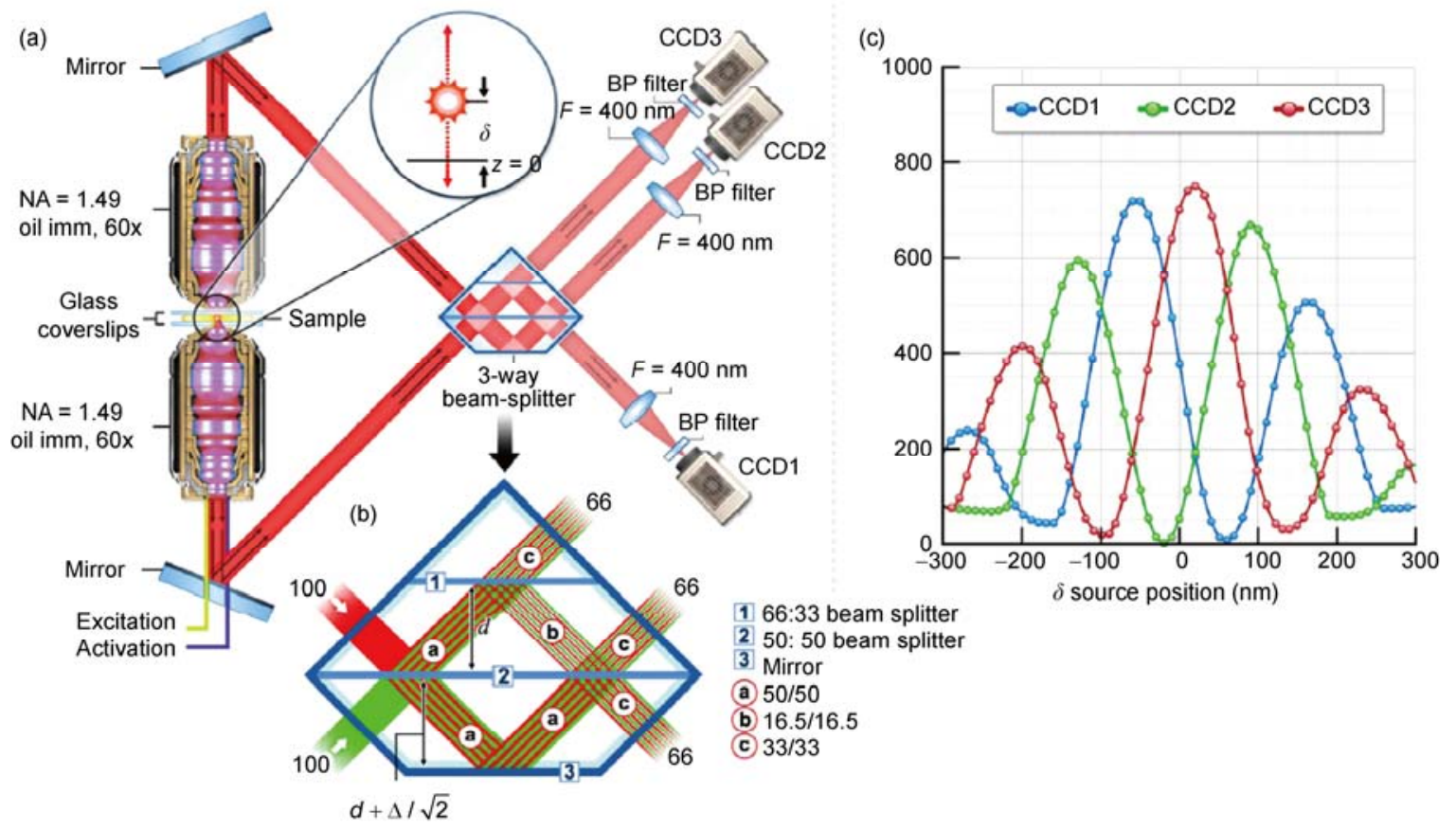

Figure 19 (Color online) The principle of iPALM. (a) The opposing lenses simultaneously collect fluorescent signals. The data is divided into three parts and propagates along three optical paths to CCD1, CCD2 and CCD3. (b) The optical density ratio and direction of propagation of the two beams at the splitters are (1) 66:33, (2) 50:50, and (3) is a mirror; (a), (b), (c) represent the optical density ratios of distinct optical paths. (c) The magnitude and phase of the signals for each CCD [76]. Image courtesy of National Academy of Sciences. 
achieved by $\mathrm{I}^{5} \mathrm{~S}$ in three dimensions is basically the best result in the area of linear optics. In theory it is possible that higher resolution can be achieved if the sample produces a non-linear effect by withstanding more optical output power.

\section{Conclusion}

Novel optical nanoscopy methods have opened a new era for biological microscopic study. With this weapon, the power of conventional fluorescence microscopic methods can be reexamined to further reveal the biological signal on a new scale. Table 1 lists the marriage of fluorescence nanoscopy methods and conventional microscopic techniques. With advances in fluorescence nanoscopy, the blanks in this table will be quickly filled, and bring novel strategies for microscopic study. The application of the above mentioned methods in biological system studies are listed in the review by Rice [56].

Commercialization is often a hallmark of the maturity of a technology. In 2007, Leica developed the Leica TCS STED and TCS STED CW systems, which highly commercialized the STED, and CW STED techniques; by the end of 2009, Nikon announced that they have signed with Harvard
University, and the University of California, to collocate the STORM and SIM systems onto Nikon microscopes; in 2010, Carl Zeiss's protrusive ELYRA series made use of PALM and SIM correlative techniques. Figure 20 lists the imaging results of some commercial systems.

Because of specific molecular imaging, fluorescence microscopy plays an important role in biological cellular imaging. However, the diffraction limit of conventional optical microscopy limited our ability to visualize sub-micron levels. Starting from the 1990s, a series of far-field microscopic methods that could reach resolutions beyond the diffraction limit were reported. In enhancing the lateral resolution, the proposed methods can be classified into three modalities based on their excitation and imaging sequences: (1) sequential excitation; (2) structured excitation; and (3) stochastic excitation. In addition, to improve the axial resolution, (1) opposing lenses and (2) light sheet illumination were proposed. To master 3-D optical nanoscopy, a series of methods were combined to expand the 2-D nanoscopy to 3 -D. The development of optical far-field nanoscopy has helped to visualize dynamic views of biochemical reaction processes, reveal fundamental principles of life activity, illuminating mechanisms of disease, as well as to develop new drugs for improved medical treatment.

Table 1 Conjugation of super-resolution optical nanoscopy methods with conventional microscopic techniques

\begin{tabular}{lll}
\hline & \multicolumn{1}{c}{ Sequential } & \multicolumn{1}{c}{ Stochastic } \\
\hline Intensity & STED [17], GSD [31], RESOLFT [33], 4Pi microscopy [70] & PALM [39], STORM [50], PAINT [57] \\
Multi-labelling & STED [13] & PALM [45], STORM [80], GSDIM[52] \\
Lifetime & GSD [31] & GSDIM [52] \\
TIRF (near field) & & PALM [39], STORM [80] \\
FRET & STED [81] & STORM \\
Multiphoton & STED [22], 4Pi microscopy [64] & \\
FCS & FCS-STED [82] & \\
\hline
\end{tabular}

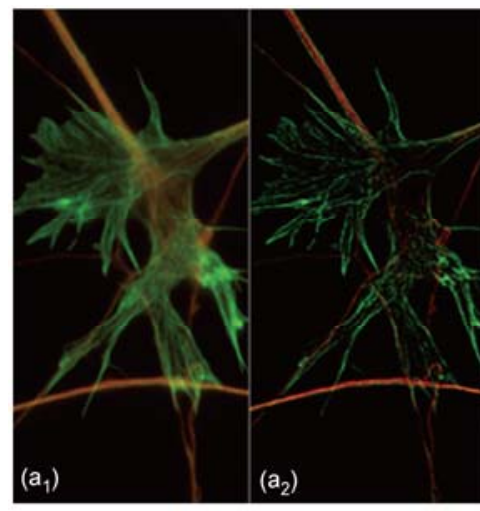

(a)



(b)

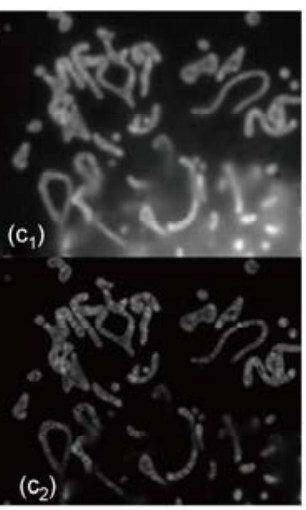

(c)

Figure 20 (Color online) (a) Neuronal images recorded by Carl Zeiss (http://www.zeiss.de/C1256A770030BCE0/WebViewAllE/3E7440D1C0672163C12576500049790A). $\left(\mathrm{a}_{1}\right)$ The result obtained from conventional wide-field microscope; $\left(\mathrm{a}_{2}\right)$ the result obtained with SR-SIM. (b) Images recorded by Leica (http://www.leica-microsystems.com/products/confocal-microscopes/details/product/leica-tcs-sted-cw-2/). ( $b_{1}$ ) The result using confocal microscopy; ( $b_{2}$ ) the result using STED. (c) Image of a NIH3T3 cell recorded by Nikon (http://www.nikon.com/about/news/2009/1203_n-sim_01.htm). (c s $_{1}$ The result using a traditional optical microscope; $\left(c_{2}\right)$ the result using N-SIM. 
The authors thank Dr. T. FitzGibbon for critical proofreading of the manuscript. This work was supported by the National Basic Research Program of China (2005CB724302, 2010CB933901), the National Natural Science Foundation of China (60588101, 60808029), the National High-Tech Research and Development Program of China (2008AA030118), Shanghai Commission of Science and Technology (064119540, 08PJ14062) and Shanghai Jiao Tong University Med-Engineering Fund (YG2009ZD201).

1 Abbe E. Beiträge zur Theorie des Mikroskops und der mikroskopischen Wahrnehmung. Arch Mikr Anat, 1873, 9: 413-418

2 Rayleigh L. On the theory of optical images with special reference to the microscope. Philos Mag, 1896, 42: 167-195

3 Born M, Wolf E. Principles of Optics. 7th ed. Cambridge: Cambridge University Press, 1999

4 Zhao N, Zhang Y H, Liu X, et al. Carbazole tricationic salt: A novel potential two-photon fluorescent DNA probe for nucleic imaging of cells. Chinese Sci Bull, 2010, 55: 3661-3667

5 Ye $\mathrm{C}$, Chen $\mathrm{C} \mathrm{Y}$, Chen $\mathrm{Z}$, et al. In situ observation of $\mathrm{C}_{60}\left(\mathrm{C}(\mathrm{COOH})_{2}\right)_{2}$ interacting with living cells using fluorescence microscopy. Chinese Sci Bull, 2006, 51: 1060-1064

6 Zhang Y, Chen S F, Ouyang Z Q, et al. Stretching and imaging studies of single DNA molecules. Chinese Sci Bull, 2000, 45: 1365-1368

7 Wang Y, Zhang J Z, Chen Y, et al. Characterization of GLUT4containing vesicles in 3T3-L1 adipocytes by total internal reflection fluorescence microscopy. Sci China Ser C-Life Sci, 2009, 52: 665671

8 Liu D H, Xiao X M, Jia R F, et al. Observation of hydrocarbon generation and migration of highly-matured carbonates by means of laser-induced fluorescence microscopy. Chinese Sci Bull, 2000, 45(Suppl): 16-20

9 Wang Z H, Jin Z, Zheng $\mathbf{J}$ A, et al. Wave-front correction of high-intensity fs laser beams by using closed-loop adaptive optics system. Sci China Ser A-Math Phys Astron, 2005, 48: 122-128

10 Bretschneider S, Eggeling C, Hell S W. Breaking the diffraction barrier in fluorescence microscopy by optical shelving. Phys Rev Lett, 2007, 98: 218103

11 Chen W X, Xiao F R, Liu L, et al. Breaking through the diffraction limit of far-field optical microscopy by stimulated emission depletion (STED). Laser Optr Prog, 2005, 42: 51-56

12 Donnert G, Keller J, Medda R, et al. Macromolecular-scale resolution in biological fluorescence microscopy. Proc Natl Acad Sci USA, 2006, 103: 11440-11445

13 Donnert G, Keller J, Wurm C A, et al. Two-color far-field fluorescence nanoscopy. Biophys J, 2007, 92: L67-L69

14 Hein B, Willig K I, Hell S W. Stimulated emission depletion (STED) nanoscopy of a fluorescent protein-labeled organelle inside a living cell. Proc Natl Acad Sci USA, 2008, 105: 14271-14276

15 Hell S W. Toward fluorescence nanoscopy. Nat Biotechnol, 2003, 21: 1347-1355

16 Hell S W. Microscopy and its focal switch. Nat Meth, 2009, 6: 24-32

17 Hell S W, Wichmann J. Breaking the diffraction resolution limit by stimulated emission: Stimulated-emission-depletion fluorescence microscopy. Opt Lett, 1994, 19: 780-782

18 Hell S W, Willig K, Dyba M, et al. Nanoscale resolution with focused light: STED and other RESOLFT microscopy concepts. In: Pawley J B, ed. Handbook of Biological Confocal Microscopy. 3rd ed. New York: Springer-Verlag, 2006. 571-579

19 Kasper R, Harke B, Forthmann C, et al. Single-molecule STED microscopy with photostable organic fluorophores. Small, 2010, 6: 1379-1384

20 Klar T A, Hell S W. Subdiffraction resolution in far-field fluorescence microscopy. Opt Lett, 1999, 24: 954-956

21 Lauterbach M. Fast STED microscopy. Doctor Dissertation. Göttingen: Max Planck Institute for Biophysical Chemistry, 2009

22 Moneron G, Hell S W. Two-photon excitation STED microscopy. Opt Expr, 2009, 17: 14567-14573

23 Rankin B R, Hell S W. STED microscopy with a $\mathrm{MHz}$ pulsed
stimulated-Raman-scattering source. Opt Expr, 2009, 17: 1567915684

24 Rankin B R, Kellner R R, Hell S W. Stimulated-emission-depletion microscopy with a multicolor stimulated-Raman-scattering light source. Opt Lett, 2008, 33: 2491-2493

25 Reuss M, Engelhardt J, Hell S W. Birefringent device converts a standard scanning microscope into a STED microscope that also maps molecular orientation. Opt Expr, 2010, 18: 1049-1058

26 Rittweger E, Han K Y, Irvine S E, et al. STED microscopy reveals crystal colour centres with nanometric resolution. Nat Phot, 2009, 3: 144-147

27 Westphal V, Rizzoli S O, Lauterbach M A, et al. Video-rate far-field optical nanoscopy dissects synaptic vesicle movement. Science, 2008, 320: 246-249

28 Wildanger D, Bückers J, Westphal V, et al. A STED microscope aligned by design. Opt Expr, 2009, 17: 16100-16110

29 Wildanger D, Rittweger E, Kastrup L, et al. STED microscopy with a supercontinuum laser source. Opt Expr, 2008, 16: 9614-9621

30 Willig K I, Harke B, Medda R, et al. STED microscopy with continuous wave beams. Nat Meth, 2007, 4: 915-918

31 Hell S W, Kroug M. Ground-state-depletion fluorscence microscopy: A concept for breaking the diffraction resolution limit. Appl Phys B, 1995, 60: 495-497

32 Rittweger E, Wildanger D, Hell S W. Far-field fluorescence nanoscopy of diamond color centers by ground state depletion. Europhys Lett, 2009, 86: 14001

33 Hofmann M, Eggeling C, Jakobs S, et al. Breaking the diffraction barrier in fluorescence microscopy at low light intensities by using reversibly photoswitchable proteins. Proc Natl Acad Sci USA, 2005, 102: 17565-17569

34 Sauer M. Reversible molecular photoswitches: A key technology for nanoscience and fluorescence imaging. Proc Natl Acad Sci USA, 2005, 102: 9433-9434

35 Gustafsson M G L. Nonlinear structured-illumination microscopy: Wide-field fluorescence imaging with theoretically unlimited resolution. Proc Natl Acad Sci USA, 2005, 102: 13081-13086

36 Heintzmann R, Jovin T M, Cremer C. Saturated patterned excitation microscopy - a concept for optical resolution improvement. J Opt Soc Am A, 2002, 19: 1599-1609

37 Kner P, Chhun B B, Griffis E R, et al. Super-resolution video microscopy of live cells by structured illumination. Nat Meth, 2009, 6: 339-342

38 Mao Z L, Wang C, Cheng Y. Superresolution far-field fluorescence bio-imaging: Breaking the diffraction barrier. Chin J Lasers, 2008, 35: 1283-1307

39 Betzig E, Patterson G H, Sougrat R, et al. Imaging intracellular fluorescent proteins at nanometer resolution. Science, 2006, 313: 1642-1645

40 Brown T A, Fetter R D, Tkachuk A N, et al. Approaches toward super-resolution fluorescence imaging of mitochondrial proteins using PALM. Methods, 2010, 51: 458-463

41 Hess S T, Girirajan T P K, Mason M D. Ultra-high resolution imaging by fluorescence photoactivation localization microscopy. Biophys J, 2006, 91: 4258-4272

42 Lidke K, Rieger B, Jovin T, et al. Superresolution by localization of quantum dots using blinking statistics. Opt Expr, 2005, 13: 70527062

$43 \mathrm{Lv} \mathrm{Z} \mathrm{J,} \mathrm{Lu} \mathrm{J} \mathrm{Z,} \mathrm{Wu} \mathrm{Y} \mathrm{Q,} \mathrm{et} \mathrm{al.} \mathrm{Introduction} \mathrm{to} \mathrm{theories} \mathrm{of} \mathrm{several}$ super-resolution fluorescence microscopy methods and recent advance in the field. Prog Biochem Biophys, 2009, 36: 1626-1634

44 Moerner W E. Single-molecule optical spectroscopy of autofluorescent proteins. J Chem Phys, 2002, 117: 10925-10937

45 Shroff H, Galbraith C G, Galbraith J A, et al. Dual-color superresolution imaging of genetically expressed probes within individual adhesion complexes. Proc Natl Acad Sci USA, 2007, 104: 20308-20313

46 Van de Linde S, Wolter S, Heilemann M, et al. The effect of photoswitching kinetics and labeling densities on super-resolution fluorescence imaging. J Biotechnol, 2010, 149: 260-266 
47 Vaziri A, Tang J, Shroff H, et al. Multilayer three-dimensional super resolution imaging of thick biological samples. Proc Natl Acad Sci USA, 2008, 105: 20221-20226

48 Huang B, Jones S A, Brandenburg B, et al. Whole-cell 3D STORM reveals interactions between cellular structures with nanometer-scale resolution. Nat Meth, 2008, 5: 1047-1052

49 Huang B, Wang W Q, Bates M, et al. Three-dimensional superresolution imaging by stochastic optical reconstruction microscopy. Science, 2008, 319: 810-813

50 Rust M J, Bates M, Zhuang X W. Sub-diffraction-limit imaging by stochastic optical reconstruction microscopy (STORM). Nat Meth, 2006, 3: 793-796

51 Heilemann M, Van de Linde S, Schüttpelz M, et al. Subdiffractionresolution fluorescence imaging with conventional fluorescent probes. Angew Chem Int Ed, 2008, 47: 6172-6176

52 Folling J, Bossi M, Bock H, et al. Fluorescence nanoscopy by groundstate depletion and single-molecule return. Nat Meth, 2008, 5: 943-945

53 Hell S W. Far-field optical nanoscopy. In: Single Molecule Spectroscopy in Chemistry, Physics and Biology: Nobel Symposium. Berlin: Springer, 2009. 365-398

54 Steinhauer C, Forthmann C, Vogelsang J, et al. Superresolution microscopy on the basis of engineered dark states. J Am Chem Soc, 2008, 130: 16840-16841

55 Moerner W E. New directions in single-molecule imaging and analysis. Proc Natl Acad Sci USA, 2007, 104: 12596-12602

56 Rice J H. Beyond the diffraction limit: Far-field fluorescence imaging with ultrahigh resolution. Mol Biosyst, 2007, 3: 781-793

57 Sharonov A, Hochstrasser R M. Wide-field subdiffraction imaging by accumulated binding of diffusing probes. Proc Natl Acad Sci USA, 2006, 103: 18911-18916

58 Wu D M, Liu Z W, Sun C, et al. Super-resolution imaging by random adsorbed molecule probes. Nano Lett, 2008, 8: 1159-1162

59 Yildiz A, Forkey J N, McKinney S A, et al. Myosin V walks hand-over-hand: Single fluorophore imaging with 1.5-nm localization. Science, 2003, 300: 2061-2065

60 Cheezum M K, Walker W F, Guilford W H. Quantitative comparison of algorithms for tracking single fluorescent particles. Biophys J, 2001, 81: 2378-2388

61 Thompson R E, Larson D R, Webb W W. Precise nanometer localization analysis for individual fluorescent probes. Biophys $\mathrm{J}$, 2002, 82: 2775-2783

62 Huang B. Super-resolution optical microscopy: Multiple choices. Curr Opin Chem Biol, 2010, 14: 10-14

63 Pertsinidis A, Zhang Y, Chu S. Subnanometre single-molecule localization, registration and distance measurements. Nature, 2010, 466: 647-651

64 Lang M C, Engelhardt J, Hell S W. 4Pi microscopy with linear fluorescence excitation. Opt Lett, 2007, 32: 259-261

65 Keller P J, Stelzer E H K. Quantitative in vivo imaging of entire embryos with digital scanned laser light sheet fluorescence micro- scopy. Curr Opin Neurobiol, 2008, 18: 624-632

66 Huisken J, Stelzer E, Swoger J. Selective plane illumination microscopy: Optical sections from many directions. GIT Lab J Eur, 2005, 9: 27-29

67 Gustafsson M G L, Agard D A, Sedat J W. I5 M: 3D widefield light microscopy with better than $100 \mathrm{~nm}$ axial resolution. J Microsc, 1999, 195: $10-16$

68 Hell S W, Lindek S, Cremer C, et al. Measurement of the 4Piconfocal point spread function proves $75 \mathrm{~nm}$ axial resolution. Appl Phys Lett, 1994, 64: 1335-1337

69 Hell S W, Stelzer E H K, Lindek S, et al. Confocal microscopy with an increased detection aperture: Type-B 4Pi confocal microscopy. Opt Lett, 1994, 19: 222-224

70 Schrader M, Hell S W. 4Pi-confocal images with axial superresolution. J Microsc, 1996, 183: 110-115

71 Gugel H, Bewersdorf J, Jakobs S, et al. Cooperative 4Pi excitation and detection yields sevenfold sharper optical sections in live-cell microscopy. Biophys J, 2004, 87: 4146-4152

72 Schrader M, Hell S W, Van der Voort H T M. Three-dimensional super-resolution with a 4Pi-confocal microscope using image restoration. J Appl Phys, 1998, 84: 4033-4042

73 Chen J, Midorikawa K. Two-color two-photon 4Pi fluorescence microscopy. Opt Lett, 2004, 29: 1354-1356

74 Schmidt R, Wurm C A, Jakobs S, et al. Spherical nanosized focal spot unravels the interior of cells. Nat Meth, 2008, 5: 539-544

75 Pavani S R P, Thompson M A, Biteen J S, et al. Three-dimensional, single-molecule fluorescence imaging beyond the diffraction limit by using a double-helix point spread function. Proc Natl Acad Sci USA, 2009, 106: 2995-2999

76 Shtengel G, Galbraith J A, Galbraith C G, et al. Interferometric fluorescent super-resolution microscopy resolves 3D cellular ultrastructure. Proc Natl Acad Sci USA, 2009, 106: 3125-3130

77 Schermelleh L, Carlton P M, Haase S, et al. Subdiffraction multicolor imaging of the nuclear periphery with 3D structured illumination microscopy. Science, 2008, 320: 1332-1336

78 Shao L, Isaac B, Uzawa S, et al. I 5 : Wide-field light microscopy with 100-nm-scale resolution in three dimensions. Biophys J, 2008, 94: 4971-4983

79 Gustafsson M G L. Surpassing the lateral resolution limit by a factor of two using structured illumination microscopy. J Microsc, 2000, 198: 82-87

80 Aaron J S, Carson B, Timlin J A. Simultaneous, dual-color STORM imaging at the cellular interface. Microsc Microanal, 2010, 16: 1112-1113

81 Auksorius E, Boruah B R, Dunsby C, et al. Stimulated emission depletion microscopy with a supercontinuum source and fluorescence lifetime imaging. Opt Lett, 2008, 33: 113-115

82 Eggeling C, Ringemann C, Medda R, et al. Direct observation of the nanoscale dynamics of membrane lipids in a living cell. Nature, 2008, 457: 1159-1162

Open Access This article is distributed under the terms of the Creative Commons Attribution License which permits any use, distribution, and reproduction in any medium, provided the original author(s) and source are credited. 